\title{
Estimating the point spread function of the adaptive optics system ADONIS using the wavefront sensor measurements
}

\author{
S. Harder and A. Chelli \\ Laboratoire d'Astrophysique, Observatoire de Grenoble, BP. 53, F-38041 Grenoble, France
}

Received July 20; accepted October 12, 1999

\begin{abstract}
Adaptive Optics (AO) has been developed to compensate the wavefront deformation due to the atmospheric turbulence. The wavefront correction, however, is often only partial and a residual blur is present in a long exposure image. It is therefore important to deconvolve the image to do accurate photometry or to detect faint structures. For this, the point spread function (PSF) has to be known. The PSF is highly variable in time and difficult to calibrate. We developed an algorithm which reconstructs the PSF of the ADONIS AO system from the wavefront sensor measurements. We present the results obtained for different observing conditions and discuss the limits of the method. We prove the existence of a local non-stationary turbulence from analyzing the spatial and temporal behaviour of the turbulent phase. We found difficult to achieve photometric precision higher than 5 to $10 \%$ on secondary components of multiple systems with flux ratio larger than 4 .
\end{abstract}

Key words: instrumentation: adaptive optics - methods: data analysis — methods: observational — techniques: image processing

\section{Introduction}

Astronomical observations on earth are strongly affected by turbulent air motions in the atmosphere. They deform randomly the wavefront of the incoming light of an astronomical source, so that randomly distributed speckles are usually present in a short-exposure image ${ }^{1}$. In a longexposure image these speckles sum up to form a blurred image. Atmospheric turbulence set therefore a severe limit to the angular resolution which, in the optical wavelength

Send offprint requests to: S. Harder

Correspondence to: Stephan.Harder@obs.ujf-grenoble.fr

${ }^{1}$ Considering observations with large apertures in the optical and infrared range. range, is rarely better than the theoretical angular resolution of a 10 to $20 \mathrm{~cm}$ aperture.

Adaptive optics (AO) has been developed to overcome this problem. It corrects the wavefront deformation in real time by means of a deformable mirror (DM) and a wavefront sensor (WFS). Due to incomplete measurements, noise and time-lag errors, the correction is often only partial and the image of a point-like source consists of a diffraction-limited peak superimposed on a large diffuse halo. It is therefore important to deconvolve the astronomical images in order to do accurate photometry or to detect faint structures without ambiguities. For this, the point spread function (PSF) of the system "atmospheretelescope" has to be known.

The classical approach consists in calibrating the PSF by taking an image of a point-like source just before and/or after the object acquisition. The quality of the wavefront correction, however, and hence the PSF too, depends on the atmospheric conditions and the magnitude of the reference star. Due to variations of the atmospheric seeing and differences in the properties of the reference sources, the observed PSF may not correspond accurately enough to the PSF at the time of the observation of the object.

In theory, this problem can be overcome when estimating the long-exposure PSF from the WFS measurements saved during the object acquisition. In this approach, however, we have to assume that the high-frequency part of the turbulent phase follows the Kolmogorov model. A method which reconstructs the PSF from WFS measurements has been developed by Véran et al. (1997) and successfully applied to the AO system PUEO installed at the Canada-France-Hawaii Telescope (CFHT). We have adapted their method to ADONIS, the AO system at the ESO $3.6 \mathrm{~m}$ telescope, which is based on a Shack-Hartmann device instead of a curvature sensor as used in the PUEO system.

The reader should keep in mind, that the reconstructed PSF will always correspond to the on-axis PSF, i.e. the PSF of the reference source. If the separation between 
the object source and the reference source is so large that anisoplanetism effects ${ }^{2}$ cannot be neglected anymore, then the reconstructed PSF will not be exactly the same as the object PSF. We investigated, if it was possible to reconstruct the on-axis PSF from the WFS measurements, and did not include anisoplanetism effects.

Section 2 recalls the concept of an AO system and analyzes the different elements taking as example the ADONIS system. We give a short description of the PSF reconstruction algorithm and refer the interested reader to Véran et al. (1997a) or Véran (1997) for a more detailed description of the method. In Sect. 3 we present the experimental approach of the reconstruction method to ADONIS. In particular, we discuss the methods of determining the measurement noise and Fried's parameter $r_{0}$. Section 4 presents the results of our PSF reconstruction obtained for different observing conditions. Finally, Sect. 5 discusses the limits of the PSF reconstruction and the photometric precision one can expect when deconvolving images with the reconstructed PSF.

\section{PSF reconstruction: Formalism}

\subsection{The adaptive optics system}

The adaptive optics system consists of three major parts: the wavefront sensor (WFS), the real-time processor and the deformable mirror $(\mathrm{DM})^{3}$. Figure 1 gives a schematic view of an AO system. The incident turbulent wavefront is corrected by the DM, then splitted into two parts. One part forms the image on a detector, the other one falls on the WFS which measures the degree of the residual wavefront deformation. A real-time processor transforms the WFS measurements into a signal which is sent to the deformable mirror to readapt the surface of the mirror to the turbulent wavefront.

\subsection{Modal description of the phase}

For astronomical applications the near-field approximation holds (Roddier 1981) which means that the amplitude of the light complex field can be considered as constant over the telescope's pupil. Hence, only the phase variations will affect the quality of the image. It is useful to express the phase $\Phi(\boldsymbol{r}, t)$ on a basis of eigenmodes $Z_{i}(\boldsymbol{r})$,

$\Phi(\boldsymbol{r}, t)=\sum_{i=1}^{\infty} z_{i}(t) Z_{i}(\boldsymbol{r})$.

\footnotetext{
${ }^{2}$ The wavefronts of the object and the reference source pass through distinct parts of the atmosphere and are therefore deformed differently.

${ }^{3}$ The deformable mirror may actually consist of several parts. E.g. a flat mirror for the tip-tilt correction and a mirror with a deformable surface to correct the higher modes. Here we will just speak of the DM.
}

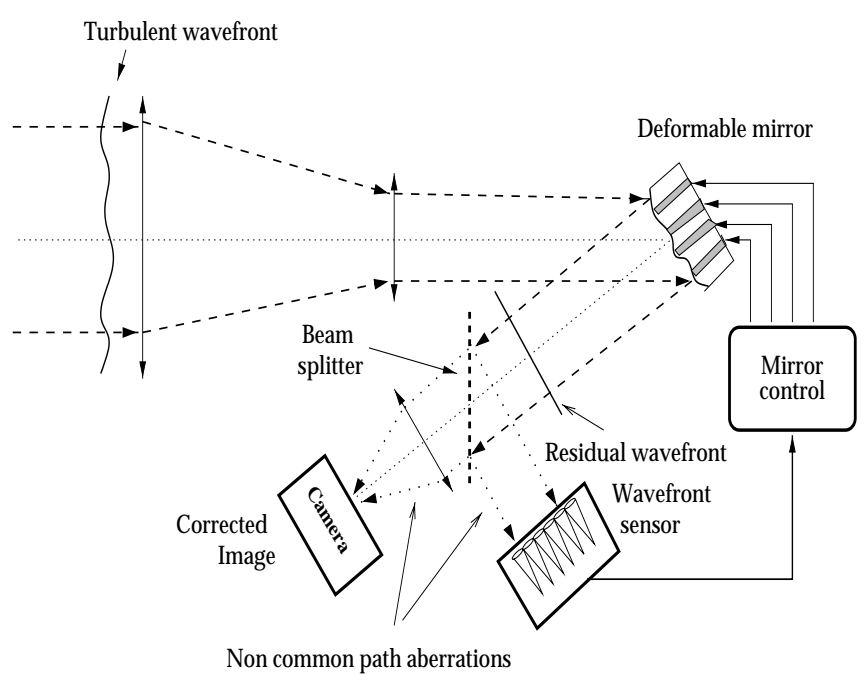

Fig. 1. Schematic view of an adaptive optics system

Table 1. Notations used in this paper

$\begin{array}{ll}\text { AO } & \text { adaptive optics } \\ \text { WFS } & \text { wavefront sensor } \\ \text { DM } & \text { deformable mirror } \\ \mathcal{M} & \text { mirror space } \\ \Phi_{\mathrm{a}}(\boldsymbol{r}, t) & \text { turbulent phase } \\ \Phi_{\mathrm{m}}(\boldsymbol{r}, t) & \text { mirror phase configuration } \\ \Phi_{\epsilon}(\boldsymbol{r}, t) & \text { residual phase } \\ \Phi_{\|}(\boldsymbol{r}, t) & \text { phase projected on } \mathcal{M} \\ \Phi_{\perp}(\boldsymbol{r}, t) & \text { phase perpendicular to } \mathcal{M} \\ N & \text { number of mirror modes } \\ \boldsymbol{w}(t)=\left\{w_{1}, \ldots, w_{\mathrm{M}}\right\} & \text { wavefront sensor measurements } \\ \boldsymbol{n}_{w}(t)=\left\{n_{w_{1}}, \ldots, n_{w_{\mathrm{M}}}\right\} & \text { WFS measurement noise } \\ \boldsymbol{a}(t)=\left\{a_{1}, \ldots, a_{N}\right\} & \text { turbulent modal coefficients } \\ \boldsymbol{\epsilon}(t)=\left\{\epsilon_{1}, \ldots, \epsilon_{N}\right\} & \text { residual modal coefficients } \\ \boldsymbol{m}(t)=\left\{m_{1}, \ldots, m_{N}\right\} & \text { modal mirror commands } \\ \boldsymbol{r}(t)=\left\{r_{1}, \ldots, r_{N}\right\} & \text { remaining error } \\ \boldsymbol{n}(t)=\left\{n_{1}, \ldots, n_{N}\right\} & \text { measurement noise projected onto } \\ & \text { the modes } \\ g_{i} \in[0,1] & \text { modal gains } \\ H_{\text {cor }}\left(g_{i}, \nu\right) & \text { correction transfer function } \\ H_{\mathrm{cl}}\left(g_{i}, \nu\right) & \text { close loop transfer function } \\ H_{\mathrm{n}}\left(g_{i}, \nu\right) & \text { noise transfer function } \\ D & \text { interaction matrix between mirror } \\ D^{+} & \text {and WFS } \\ \mathcal{W} & \text { control matrix } \\ & \text { operator describing the WFS }\end{array}$

The most common basis of modes are the Zernike polynomials (Noll 1976) and the Karhunen-Loeve functions (Wang \& Markey 1978). Another basis of eigenfunctions is obviously the basis of mirror modes which of course is finite and can therefore only express the low-frequency part of the turbulent phase (for frequencies typically less than the inverse of the distance between two actuators). We will call this basis $\mathcal{M} . \Phi_{\mathrm{m}}(\boldsymbol{r}, t)$ is the phase function 
generated by the mirror commands $m_{i}(t)$ and the mirror modes $M_{i}(\boldsymbol{r})$ :

$\phi_{\mathrm{m}}(\boldsymbol{r}, t)=\sum_{i=1}^{N} m_{i}(t) M_{i}(\boldsymbol{r}) \quad M_{i}(\boldsymbol{r}) \in \mathcal{M}$,

where $N$ is the number of the mirror modes. The residual phase, the phase after the AO correction, is given by:

$\Phi_{\epsilon}(\boldsymbol{r}, t)=\Phi_{\epsilon_{\|}}(\boldsymbol{r}, t)+\Phi_{\mathrm{a}_{\perp}}(\boldsymbol{r}, t)$,

where

$\Phi_{\epsilon_{\|}}(\boldsymbol{r}, t)=\Phi_{\mathrm{a}_{\|}}(\boldsymbol{r}, t)-\Phi_{\mathrm{m}}(\boldsymbol{r}, t)$

is the low-frequency part of the turbulent phase $\phi_{\mathrm{a}}(\boldsymbol{r}, t)$, partially corrected by the system:

$\phi_{\epsilon_{\|}}(\boldsymbol{r}, t)=\sum_{i=1}^{N} \epsilon_{i}(t) M_{i}(\boldsymbol{r})$

and $\Phi_{\mathrm{a}_{\perp}}(\boldsymbol{r}, t)$ is the high-frequency part of the turbulent phase which is not affected by the AO correction at all. Let $\phi_{\mathrm{a} \|}(\boldsymbol{r}, t)$ be the projection of $\phi_{\mathrm{a}}(\boldsymbol{r}, t)$ onto $\mathcal{M}$ :

$\phi_{\mathrm{a}_{\|}}(\boldsymbol{r}, t)=\sum_{i=1}^{N} a_{i}(t) M_{i}(\boldsymbol{r})$.

We can express $\Phi_{\epsilon_{\|}}(\boldsymbol{r}, t)$ in vector form:

$\boldsymbol{\epsilon}_{\|}(t)=\boldsymbol{a}_{\|}(t)-\boldsymbol{m}(t)$

where $\boldsymbol{\epsilon}=\left\{\epsilon_{1}, . ., \epsilon_{N}\right\}, \boldsymbol{a}=\left\{a_{1}, . ., a_{N}\right\}$ and $\boldsymbol{m}=\left\{m_{1}, . ., m_{N}\right\}$. For a "perfect" correction, we would have $\boldsymbol{\epsilon}_{\|}=\mathbf{0}$. Table 1 summaries the notations used in this paper.

The ADONIS system possesses two mirrors for the wavefront correction: a plan mirror for the tip/tilt correction and a deformable mirror with 52 actuators (piezostack elements). Due to invisible and redundant modes, only 50 modes are corrected (Gendron \& Léna 1994).

\subsubsection{The wavefront sensor}

The wavefront sensor (WFS) of the ADONIS system is a Shack-Hartmann device. It is a grid of $7 \times 7$ sub-lenses placed in the conjugate plane of the telescope pupil. Due to the partial shielding by the secondary mirror, the system uses only 32 out of 49 sub-apertures. Each lens forms a spot on a detector whose location $\boldsymbol{w}(t)$ depends on the average phase gradient over the sub-aperture,

$\boldsymbol{w}(t) \propto \int_{\text {sub }} \nabla \Phi_{\epsilon}(\boldsymbol{r}, t) \mathrm{d} \boldsymbol{r}$.

The relationship between the WFS measurements $\boldsymbol{w}(t)$ and $\Phi_{\epsilon}(\boldsymbol{r}, t)$ is supposed to be linear. The interaction matrix $D$ describes the relationship between the low-order modal coefficients $\boldsymbol{\epsilon}$ and the slope measurements $\boldsymbol{w}(t)$. The expression for the WFS measurements is then given by:

$\boldsymbol{w}(t)=D \boldsymbol{\epsilon}(t)+\mathcal{W}\left[\phi_{\mathrm{a}_{\perp}}(\boldsymbol{r}, t)\right]+\boldsymbol{n}_{w}(t)$.
The first term of the right-hand side describes the WFS measurements due to the low-order residual phase $\boldsymbol{\epsilon}(t)$, the second term is the contribution of the high-order noncorrected phase $\Phi_{\mathrm{a}_{\perp}}(\boldsymbol{r}, t)$ to the WFS measurements and the last term is the measurement noise. The symbol $\mathcal{W}$ stands for the operator describing the WFS. ADONIS actually uses two WFS cameras, the RETICON for high-flux sources ( $\leq 8$ magnitudes) and the EBCCD for low-flux sources (8-13 magnitudes).

\subsubsection{The control loop}

From the WFS measurements $\boldsymbol{w}(t)$, the low-order residual phase estimate $\widehat{\boldsymbol{\epsilon}}(t)$ is calculated from a least-square fit of the equation $\boldsymbol{w}(t)=D \boldsymbol{\epsilon}(t)$, which leads to:

$\widehat{\boldsymbol{\epsilon}}(t)=D^{+} \boldsymbol{w}(t)$,

where $D^{+}$is the generalized inverse of $D$, i.e. the control matrix,

$D^{+}=\left(D^{T} D\right)^{-1} D^{T}$.

Using the Eqs. (9) and (10) we find the following relation:

$\widehat{\boldsymbol{\epsilon}}(t)=\boldsymbol{\epsilon}(t)+\boldsymbol{r}(t)+\boldsymbol{n}(t)$,

where $\boldsymbol{r}(t)$ is the modal error due to the contribution of the high-order phase to the WFS measurements, which is interpreted as a combination of spatial aliasing and crosscorrelation (Hermann 1981; Southwell 1982):

$\boldsymbol{r}(t)=D^{+} \mathcal{W}\left[\phi_{\perp}(\boldsymbol{r}, t)\right]$.

We call it remaining error (Dai 1996). $\boldsymbol{n}(t)$ is the WFS measurement noise propagated on the modes:

$\boldsymbol{n}(t)=D^{+} \boldsymbol{n}_{w}(t)$.

If the contribution of the noise and the high-order phase to the WFS measurements would be zero, then $\widehat{\boldsymbol{\epsilon}}(t)=\boldsymbol{\epsilon}(t)$, and the new mirror commands would be $\boldsymbol{m}(t+\tau)=$ $\boldsymbol{m}(t)+\widehat{\boldsymbol{\epsilon}}(t)$. Since this is not the case, the components of $\widehat{\boldsymbol{\epsilon}}(t)$ are multiplied by a gain $g$ with values between 0 and 1 in order to reduce the error contribution to the residual phase variance. While the zonal correction consists in taking the same gain for all modes, the modal control, as applied in the ADONIS system, determines a specific gain for each mode (Gendron \& Léna 1994). This has the advantage to take into account that the relative magnitude between the variance of the turbulent coefficients $\sigma_{\mathrm{a}_{i}}^{2}$ and the modal noise $\sigma_{\mathrm{n}_{i}}^{2}$ is different for each mode.

The following equation will help to understand the modal control. It describes the wavefront correction in the Fourier domain (Gendron \& Léna 1994):

$$
\begin{aligned}
\widetilde{\epsilon_{i}}(\nu)= & H_{\mathrm{cor}}\left(g_{i}, \nu\right) \widetilde{a_{i}}(\nu)-H_{\mathrm{cl}}\left(g_{i}, \nu\right) \widetilde{r_{i}}(\nu) \\
& -H_{\mathrm{n}}\left(g_{i}, \nu\right) \widetilde{n_{i}}(\nu),
\end{aligned}
$$

where $H_{\mathrm{cor}}, H_{\mathrm{cl}}$ and $H_{\mathrm{n}}$ are the correction transfer function, the close loop transfer function and the noise transfer function, respectively. The noise transfer function is 


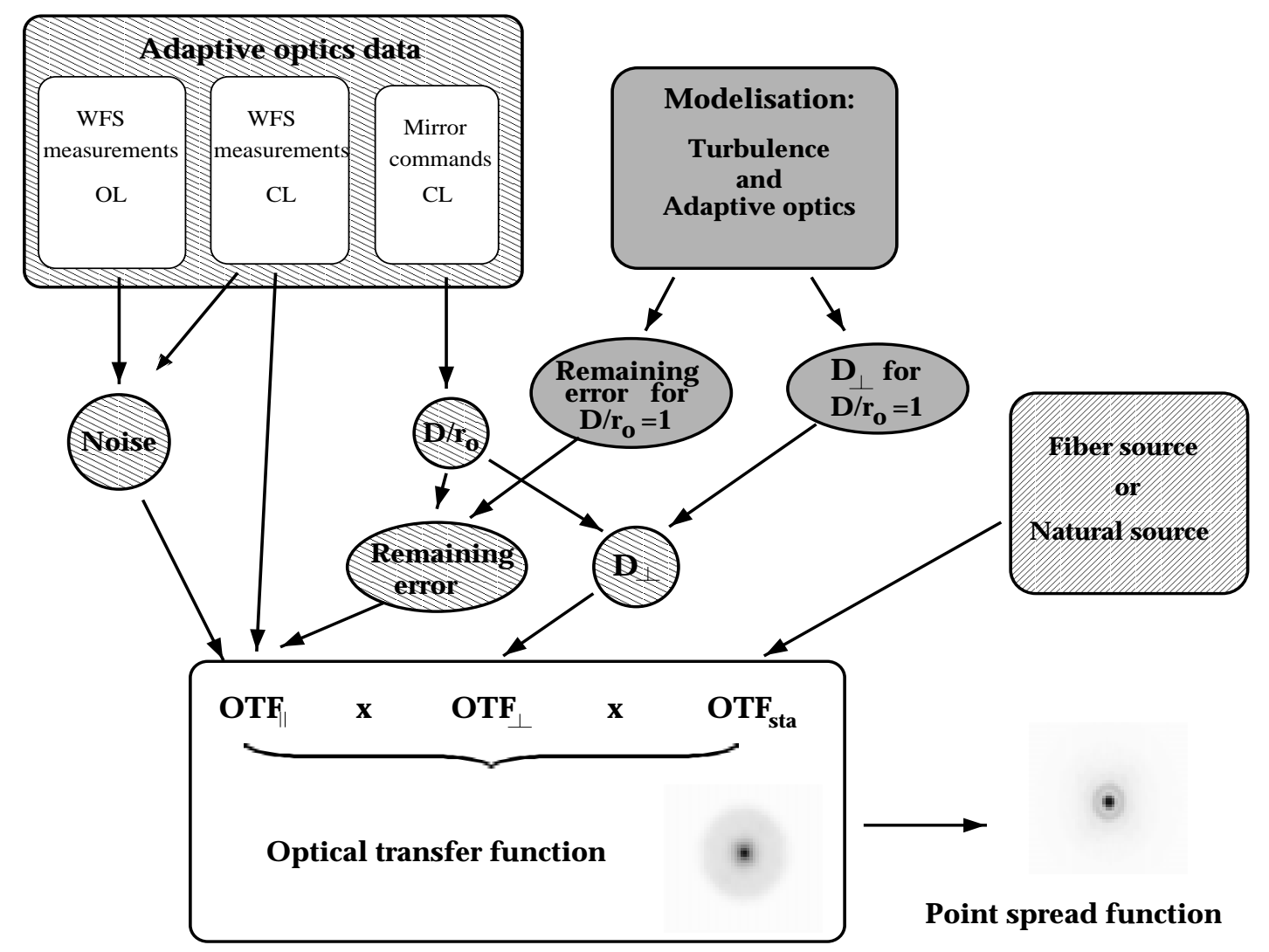

updated at each object acquisition

Fig. 2. Scheme of the PSF reconstruction method

similar to the close loop transfer function which is a lowpass frequency filter. The correction transfer function is a high-pass frequency filter equal to: $H_{\mathrm{cor}}=1-H_{\mathrm{cl}}$. The system bandwidth is defined by the frequency for which $\left|H_{\mathrm{cl}}\right|$ drops beneath $-3 \mathrm{~dB}$. It corresponds roughly to the lowest frequency transmitted by $\left|H_{\text {cor }}\right|$ and decreases as the gain is reduced. Hence, for high gains, $H_{\text {cor }}$ reduces efficiently the turbulent modes $a_{i}$, while the remaining error $r_{i}$ and the noise contribution $n_{i}$ are not filtered. In order to reduce their contribution, we have to decrease the gains. But then, the turbulent modes are less corrected. The modal control consists in determining for each mode the gain which minimizes both the contribution from the turbulent modes and the contribution from the remaining error and the measurement noise.

\subsection{The long-exposure image}

The partially corrected long-exposure optical transfer function (OTF) in adaptive optics is given by (Conan 1995; Véran 1997)

$$
\begin{aligned}
\langle\mathrm{OTF}(\boldsymbol{\rho} / \lambda)\rangle= & \frac{1}{S} \int\left\langle\mathrm{e}^{i \phi_{\epsilon}(\boldsymbol{r}, t)} \mathrm{e}^{-i \phi_{\epsilon}(\boldsymbol{r}+\boldsymbol{\rho}, t)}\right\rangle \times \\
& P_{\mathrm{o}}(\boldsymbol{r}) P_{\mathrm{o}}(\boldsymbol{r}+\boldsymbol{\rho}) \mathrm{d} \boldsymbol{r},
\end{aligned}
$$

where $S$ is the pupil's surface area and $P_{\mathrm{o}}(\boldsymbol{r})$ is the complex pupil function which is $\mathrm{e}^{i \phi_{0}(\boldsymbol{r})}$ inside the pupil and 0 otherwise. The term $\phi_{0}(\boldsymbol{r})$ represents static aberrations not corrected by the AO system. They are essentially lowfrequency aberrations which arise after the splitting of the light beam, either in the optical path of the science camera or in the optical path of the WFS. Some hypotheses are introduced to simplify the last equation. If $\phi_{\epsilon}(\boldsymbol{r}, t)$ is a random variable of Gaussian statistics, we have the following relation:

$\left\langle\mathrm{e}^{i \phi_{\epsilon}(\boldsymbol{r}, t)} \mathrm{e}^{-i \phi_{\epsilon}(\boldsymbol{r}+\boldsymbol{\rho}, t)}\right\rangle=\mathrm{e}^{-\frac{1}{2} D_{\phi_{\epsilon}}(\boldsymbol{r}, \boldsymbol{\rho})}$,

where

$D_{\phi_{\epsilon}}(\boldsymbol{r}, \boldsymbol{\rho})=\left\langle\left[\phi_{\epsilon}(\boldsymbol{r}, t)-\phi_{\epsilon}(\boldsymbol{r}+\boldsymbol{\rho}, t)\right]^{2}\right\rangle$

is the phase structure function of $\phi_{\epsilon}(\boldsymbol{r}, t)$. The phase structure function depends on $\boldsymbol{r}$. We will replace it by $\bar{D}_{\phi_{\epsilon}}(\boldsymbol{\rho})$, its average over the pupil's aperture (Conan 1995; Véran et al. 1997a). Then, we can write:

$\langle\mathrm{OTF}(\boldsymbol{\rho} / \lambda)\rangle=\mathrm{e}^{-\frac{1}{2} \bar{D}_{\phi_{\epsilon}}(\boldsymbol{\rho})} \mathrm{OTF}_{\text {sta }}(\boldsymbol{\rho} / \lambda)$.

The last term of this equation is the instrumental optical transfer function,

$\mathrm{OTF}_{\mathrm{sta}}(\boldsymbol{\rho} / \lambda)=\frac{1}{S} \int P_{\mathrm{o}}(\boldsymbol{r}) P_{\mathrm{o}}(\boldsymbol{r}+\boldsymbol{\rho}) \mathrm{d} \boldsymbol{r}$, 
which can, for example, be calibrated by measuring an artificial source in close loop.

Since $\phi_{\epsilon}(\boldsymbol{r}, t)=\phi_{\epsilon_{\|}}(\boldsymbol{r}, t)+\phi_{\mathrm{a}_{\perp}}(\boldsymbol{r}, t)$, we can write the mean phase structure function as:

$\bar{D}_{\phi_{\epsilon}}(\boldsymbol{\rho})=\bar{D}_{\phi_{\epsilon_{\|}}}(\boldsymbol{\rho})+\bar{D}_{\phi_{\mathrm{a}_{\perp}}}(\boldsymbol{\rho})+\Gamma_{\phi_{\epsilon_{\|}} \phi_{\mathrm{a}_{\perp}}}(\boldsymbol{\rho})$

where $\bar{D}_{\phi_{\epsilon_{\|}}}$is the mean structure function of $\phi_{\epsilon_{\|}}, \bar{D}_{\phi_{\mathrm{a}_{\perp}}}$ the mean structure function of $\phi_{\mathrm{a}_{\perp}}$ and $\Gamma_{\phi_{\epsilon_{\|}} \phi_{\mathrm{a}_{\perp}}}$ a crossed term which we will neglect in our calculation (Véran et al. 1997a). The long-exposure optical transfer function can then be written as the product of three terms:

$<\operatorname{OTF}\left(\frac{\boldsymbol{\rho}}{\lambda}\right)>=\mathrm{e}^{-\frac{1}{2} \bar{D}_{\phi_{\epsilon}}(\boldsymbol{\rho})} \mathrm{e}^{-\frac{1}{2} \bar{D}_{\phi_{a_{\perp}}}(\boldsymbol{\rho})} \operatorname{OTF}_{\text {sta }}\left(\frac{\boldsymbol{\rho}}{\lambda}\right)$.

2.3.1. Estimation of the low-order phase structure function

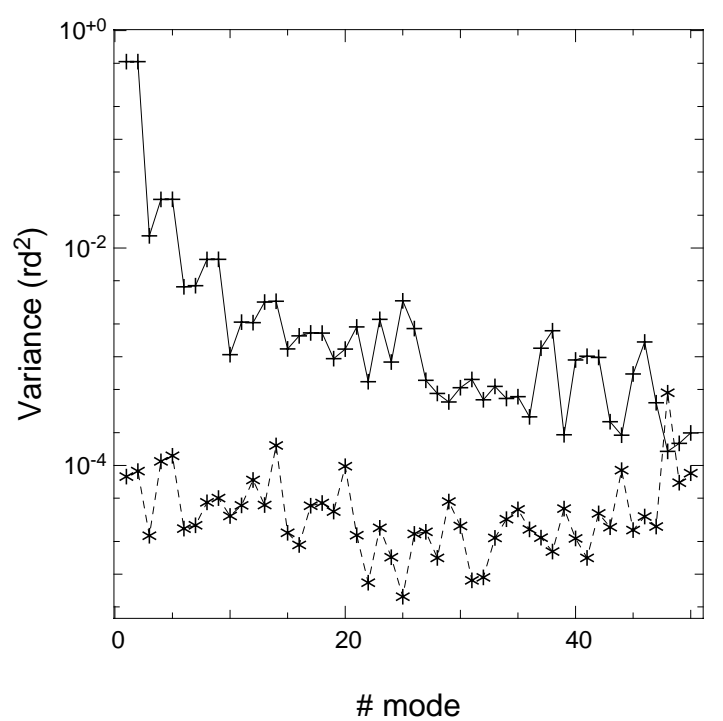

Fig. 3. Variance of the turbulent mode coefficients $\sigma_{\mathrm{a}_{i}}^{2}$ (continuous line) and variance of the remaining error $\sigma_{\mathrm{r}_{i}}^{2}$ (dashed line) versus the mode number $i$, assuming Kolmogorov turbulence and $D / r_{0}=1$

We estimate $\bar{D}_{\phi_{\epsilon_{\|}}}(\boldsymbol{\rho})$ from:

$\bar{D}_{\phi_{\epsilon_{\|}}}(\boldsymbol{\rho})=\sum_{i, j=1}^{N}<\epsilon_{i} \epsilon_{j}>U_{i j}(\boldsymbol{\rho})$,

where

$U_{i j}(\boldsymbol{\rho})=$

$\frac{\int\left[M_{i}(\boldsymbol{r})-M_{i}(\boldsymbol{r}+\boldsymbol{\rho})\right]\left[M_{j}(\boldsymbol{r})-M_{j}(\boldsymbol{r}+\boldsymbol{\rho})\right] P(\boldsymbol{r}) P(\boldsymbol{r}+\boldsymbol{\rho}) \mathrm{d} \boldsymbol{r}}{\int P(\boldsymbol{r}) P(\boldsymbol{r}+\boldsymbol{\rho}) \mathrm{d} \boldsymbol{r}}$.

$P(\boldsymbol{r})$ is the ideal pupil function taking the value 1 inside the aperture and 0 outside. The covariance of the residual mode coefficients $\epsilon_{i}$, found with the help of Eqs. (12) and (15) and after some calculation, is given by:

$$
\begin{aligned}
<\epsilon_{i} \epsilon_{j}>= & <\hat{\epsilon}_{i} \hat{\epsilon}_{j}>-<n_{i} n_{j}>+<r_{i} r_{j}> \\
& -\int H_{\text {cor }}^{*}\left(g_{i}, \nu\right)\left[S_{\mathrm{a}_{i} \mathrm{r}_{j}}(\nu)+S_{\mathrm{r}_{i} \mathrm{r}_{j}}(\nu)\right] \mathrm{d} \nu \\
& -\int H_{\text {cor }}^{*}\left(g_{j}, \nu\right)\left[S_{\mathrm{a}_{j} \mathrm{r}_{i}}(\nu)+S_{\mathrm{r}_{i} \mathrm{r}_{j}}(\nu)\right] \mathrm{d} \nu
\end{aligned}
$$

where $S_{x x}$ represents the power spectrum of the random variable $x$ and $S_{x y}$ the cross power spectrum of the random variables $x$ and $y . H_{\text {cor }}$ is a high-pass frequency filter. Thus, if we assume that the AO bandwidth is higher than $\nu_{\mathrm{c}}$, the highest cut-off frequency of the temporal power spectra of the turbulent mirror modes, we can neglect the two last terms in Eq. (25) (Véran et al. 1997a) and get:

$\mathcal{C}_{\epsilon \epsilon}=\mathcal{C}_{\hat{\epsilon} \hat{\epsilon}}-\mathcal{C}_{n n}+\mathcal{C}_{r r}$,

where $\mathcal{C}$ stands for the covariance matrix. We determine the covariance matrix $\mathcal{C}_{\hat{\epsilon} \hat{\epsilon}}$ in Eq. (26) from the WFS measurements $\boldsymbol{w}$ and the control matrix $D^{+}, \mathcal{C}_{n n}$ from the measurement noise and $D^{+}$, and $\mathcal{C}_{r r}$ by simulation of the adaptive optics system in the presence of Kolmogorov turbulence. For this, we use a program written by F. Rigaut (Rigaut et al. 1994). To fix the ideas, Fig. 3 shows, as dashed line, the variance of the remaining error $\sigma_{\mathrm{r}_{i}}^{2}$ compared to the variance of the turbulent mode coefficients $\sigma_{\mathrm{a}_{i}}^{2}$ for a Kolmogorov turbulence, both for $D / r_{0}=1$. The total variance of the remaining error is

$\sum \sigma_{\mathrm{r}_{i}}^{2} \approx 0.0025\left(\frac{D}{r_{0}}\right)^{5 / 3}$,

where $D$ is the diameter of the telescope (be careful not to confuse with the interaction matrix $D$ ) and $r_{0}$ is Fried's parameter.

\subsubsection{Estimation of the high-order phase structure} function

With this AO simulation package we also estimate the high-order phase structure function $\bar{D}_{\phi_{\mathrm{a}}}$ for $D / r_{0}=1$. The actual phase structure function has then to be calibrated by $\left(D / r_{0}\right)^{5 / 3}$. For large distances, $\bar{D}_{\phi_{\mathrm{a}_{\perp}}}$ saturates to $2 \sigma_{\mathrm{a}_{\perp}}^{2}$ with:

$\sigma_{\mathrm{a}_{\perp}}^{2} \approx 0.012\left(\frac{D}{r_{0}}\right)^{5 / 3}$.

The scheme in Fig. 2 summarizes the PSF reconstruction process.

\section{PSF reconstruction: Experimental approach}

To test the PSF reconstruction algorithm, we measured the residual wavefront on a bright point source which is simultaneously imaged on the scientific detector. The reconstructed PSF from the WFS measurements can then be 


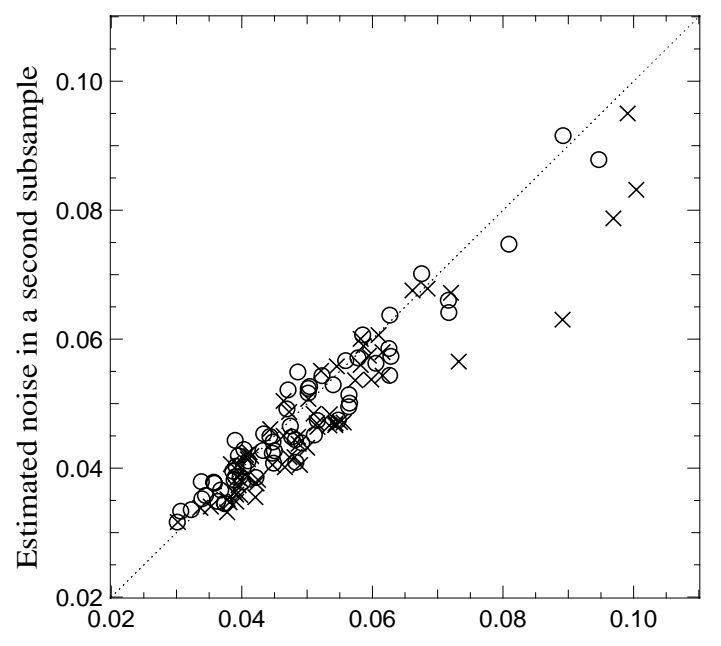

Estimated noise in one subsample

Fig. 4. Noise estimated on one set of data as a function of the noise estimated on another set of data. The rms deviation with respect to the dotted straight line (noise equality) is about $7-8 \%$

directly compared to the long-exposure image. To simulate reference sources of different magnitude, we put appropriated neutral densities in front of the WFS. For each neutral density, we took measurements alternatively in open loop $(\mathrm{OL})$ and in close loop $(\mathrm{CL})$, to compare the derived $r_{0}$ and noise values (see below).

To reconstruct the PSF, we need to know: i) the measurement noise (noise hereafter) in order to estimate accurately the residual phase covariance $\mathcal{C}_{\epsilon \epsilon}$, and ii) $r_{0}$ in order to estimate $D_{\phi_{\mathrm{a}_{\perp}}}$ and $\mathcal{C}_{r r}$. Let us first discuss the methods we use to estimate these two quantities.

\subsection{Evaluation of the noise}

The noise for a Shack-Hartmann device is generally a superposition of photon noise and read-out noise. Although theoretical expressions can be found in the literature (Rousset 1994 e.g.), it is always preferable to estimate the noise directly on the experimental data. The noise which is supposed to be white can be found from the measured wavefront slopes, either analyzing their temporal autocorrelation or their temporal spectral density. Since one is the Fourier transform of the other, both methods are in principle equivalent. In practice, we generally use the autocorrelation method which consists in deriving the noise from the bias on the zero point of the autocorrelation function by fitting e.g. a parabola near its origin (Gendron \& Léna 1995). We checked that the noise derived this way is consistent with the noise extracted from the high frequency tail of the temporal spectral density. In order to
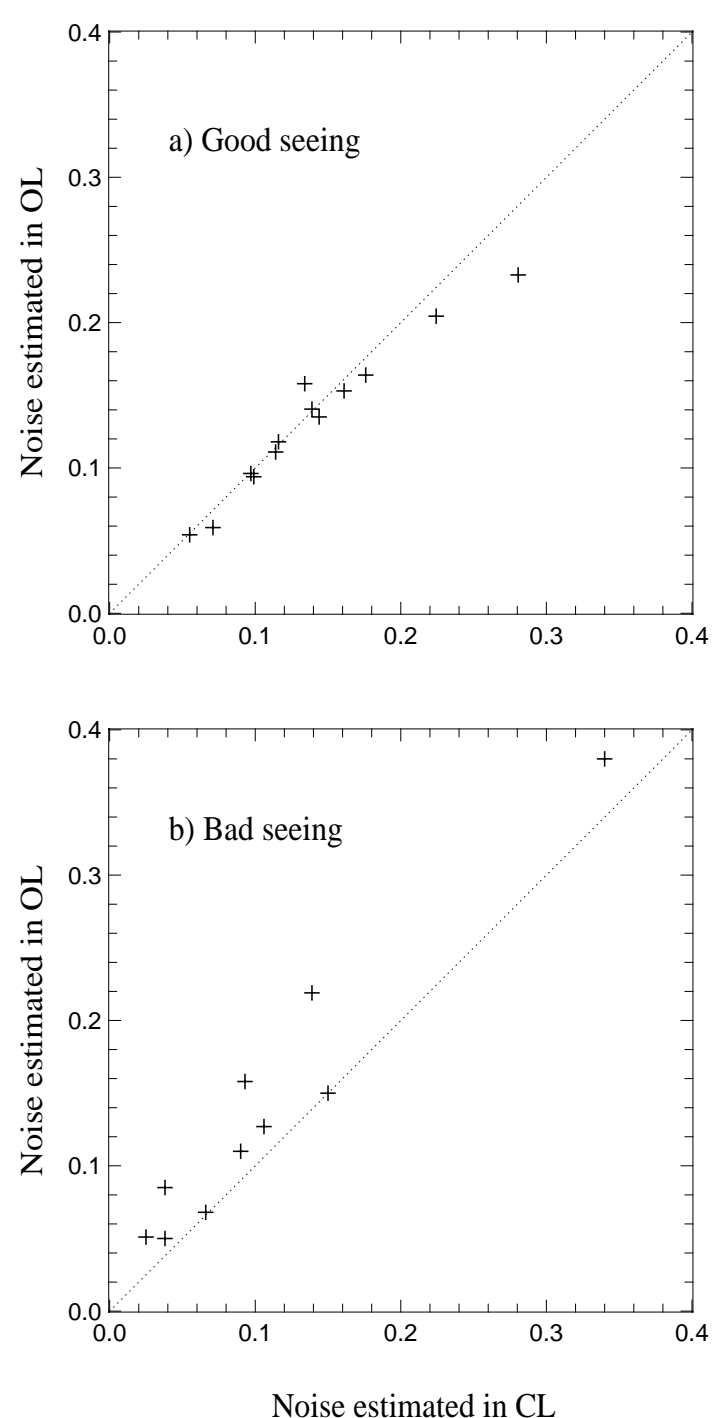

Fig. 5. Noise estimated on OL data versus the noise estimated on CL data, both averaged over all subapertures for a) good seeing $(0.8$ arcsec $)$ and b) bad seeing $(1.5-2 \operatorname{arcsec})$

test the accuracy of the method, we estimated the noise on four consecutive samples of 5120 measurements each. Figure 4 compares, for the set of useful subpupils and the $x$ and $y$ directions, the noise estimated in one sample to the noise found on another sample. The rms deviation is typically of the order of 7 to $8 \%$.

It is straightforward to estimate the noise on OL data, but it is usually very difficult to do it on CL data when the correction is good. The reason is that the consecutive slope measurements are less correlated due to the wavefront correction, making difficult to extrapolate the autocorrelation function to its origin. We estimate the noise on CL data whenever it is possible, otherwise we do it on the OL data. It is therefore important to know, if both derivations are compatible. In Fig. 5, we compare, for different set of data, the noise evaluated in OL to the noise evaluated in CL (each averaged over all subapertures), when 
this was possible, i.e. for small AO loop gains. Clearly, both derivations are consistent for good seeing $(0 . \prime 9)$. It is not quite the case for bad seeing $\left(1^{\prime \prime} .5-2^{\prime \prime} .0\right)$, for which the OL noise tends to be larger than the CL noise, probably due to the finite size of each sub-aperture. In fact, the optical field of one sub-aperture which is needed to image a focal spot accurately is of the order of $2 \mathrm{FWHM}+6 \sigma_{\alpha}$, where FWHM is the full width at half maximum of the spot and $\sigma_{\alpha}^{2}$ the variance of the angle-of-arrival. Taking the above seeing values, we find a necessary field of $\approx 77^{\prime \prime} 0$ in the case of very bad seeing and $\approx 33^{\prime \prime} 0$ in the case of good seeing. Since the field of view of the ADONIS wavefront sensor is $6{ }^{\prime \prime} 0$ (EBCCD), the image can be truncated when the seeing is bad, leading to a bad estimate of its center of gravity.

\subsection{Evaluation of Fried's parameter $r_{0}$}

We have to know $r_{0}$ in order to calibrate the highfrequency phase structure function $\bar{D}_{\phi_{\perp}}(\boldsymbol{\rho})$ and the covariance of the remaining error $\mathcal{C}_{r r}$. We now discuss the different methods to estimate $r_{0}$.

In OL, we can derive $r_{0}$ either from the variance of the absolute angle-of-arrival $\alpha$ or from the variance of the differential angle-of-arrival $\Delta \alpha_{\mathrm{d}}=\alpha(x)-\alpha(x+d)$, where $d$ is the distance between the centers of two subapertures (Ziad 1993). The advantage of using the differential angleof-arrival is that it does not depend on telescope vibrations which could bias the $r_{0}$ evaluation when using the absolute angle-of-arrival. Experimentally, we find, however, that both methods give the same results within $5 \%$.

In CL the variance of the angle-of-arrival is reduced due to the wavefront correction and hence, it cannot be directly linked to $r_{0}$. In this case, we estimate $r_{0}$ from the variance of the mirror commands by means of the following equation (Véran et al. 1997a):

$\sigma_{\mathrm{m}_{i}}^{2}=\left(\sigma_{\mathrm{a}_{0_{i}}}^{2}+\sigma_{\mathrm{r}_{0_{i}}}^{2}\right)\left(\frac{D}{r_{0}}\right)^{5 / 3}+\sigma_{n}^{2} \int\left|H_{\mathrm{n}}\left(g_{i}, \nu\right)\right| \mathrm{d} \nu$,

where $\sigma_{\mathrm{a}_{0_{i}}}^{2}$ and $\sigma_{\mathrm{r}_{0_{i}}}^{2}$ are the theoretical variances of the turbulent modal coefficients and the remaining error for $D / r_{0}=1$, respectively (assuming Kolmogorov turbulence). The last term is the modal noise filtered by the noise transfer function. Its influence on the calculation of $r_{0}$ is negligible. Also, $\sigma_{\mathrm{r}_{0}}^{2}$ is small compared to $\sigma_{\mathrm{a}_{0}}^{2}$. The above equation is valid, if the bandwidth of the AO system is higher than the highest cut-off frequency of the temporal spectra of $a_{i}$ and $r_{i}$. If this is not true, then we will overestimate $r_{0}$. The presence of an outer turbulence scale $L_{0}$ will decrease the variance of the first modes, particularly the tip/tilt modes. For this reason, we exclude these two modes. We also exclude the higher modes for which the $\mathrm{AO}$ bandwidth is low (due to small gains) and, in any case, much lower than their cut-off frequency. Thus, we restrict the estimation of $r_{0}$ to the modes 3 to 25 .

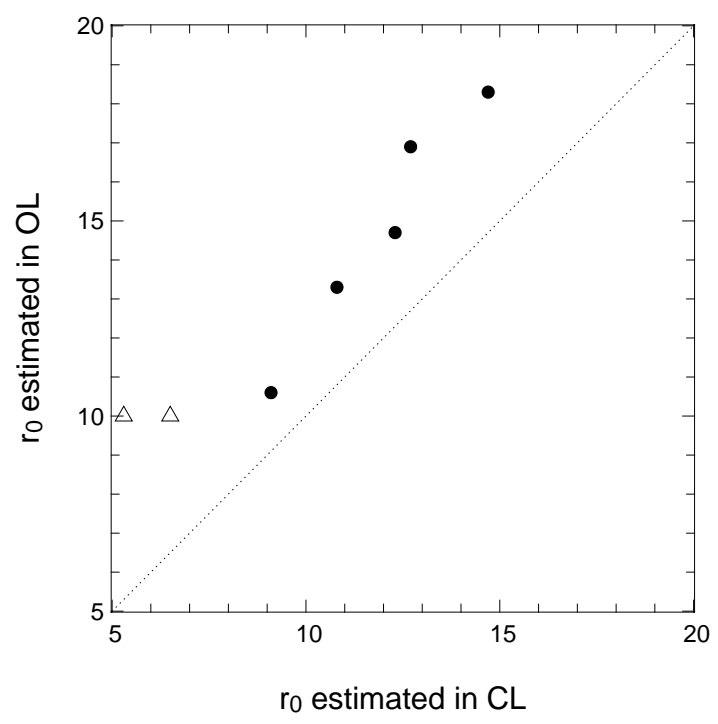

Fig. 6. $r_{0}$ estimated from the OL data (angle-of-arrival) versus the $r_{0}$ computed from the CL data (mirror commands). The two smallest $r_{0}$ values (triangles) calculated in OL are probably affected by large biases. For the larger $r_{0}$ (black circles), the values calculated from the angle-of-arrival are in good agreement with those derived from a seeing monitor located outside of the telescope dome

Figure 6 compares the values of $r_{0}$ obtained from the variance of the angle-of-arrival in OL to those obtained from the variance of the mirror commands in CL. $r_{0}$ estimated from the OL data is always larger than $r_{0}$ computed from the CL data. The two smallest $r_{0}$ values (triangles) calculated in OL are probably affected by large biases. For the larger $r_{0}$ (black circles), the values calculated from the angle-of-arrival are in good agreement with those derived from a seeing monitor located outside of the telescope dome. Theses values are in turn in good agreement with those derived from the mirror commands, if we restrict the evaluation of $r_{0}$ to the modes 3 to 5 . This indicates the possible presence of dome or mirror seeing (see Sect. 5.2).

For our PSF reconstruction, we use in practice the $r_{0}$ value obtained from the variance of the mirror commands on the modes 3 to 25 .

\section{PSF reconstruction: Results}

We present now the results of our reconstruction algorithm applied for different observing conditions:

- bright reference sources under good seeing conditions $(0.9-1$ arcsec $)$;

- faint reference sources under very bad seeing conditions $(1.5-2$ arcsec);

- faint reference sources under very good seeing conditions $(0.6-0.7$ arcsec $)$.

Table 2 summarizes the experimental conditions and the results of the PSF reconstruction. For each case, Figs. 7 
Table 2. Experimental conditions and results of the PSF reconstruction. The sampling frequency of the WFS was $200 \mathrm{~Hz}$ in all cases and its effective wavelength about $0.6 \mu \mathrm{m}$. We show $r_{0}$ estimated on the modes 3 to 25 and, in parentheses, $r_{0}$ estimated from the variance of the angle-of-arrival in $\mathrm{OL}$

\begin{tabular}{|c|c|c|c|c|c|c|c|}
\hline & Ex. A1 & Ex. A2 & Ex. B1 & Ex. B2 & Ex. C1 & Ex. C2 & Ex. C3 \\
\hline$M_{V}$ (magnitudes) & 7.4 & 7.7 & 10.3 & 10.3 & 10.6 & 11.6 & 8.5 \\
\hline Mean flux ( $\mathrm{e}^{-} / \operatorname{ssp} /$ frame $)$ & 80 & 48 & 40 & 39 & 7 & 4.5 & 25 \\
\hline$r_{0}$ at $0.55 \mu \mathrm{m}(\mathrm{cm})$ & $10.8(13.3)$ & $9.1(10.6)$ & $6.5(10.0)$ & $5.3(10.0)$ & $12.3(14.7)$ & $14.7(18.3)$ & $12.7(16.9)$ \\
\hline WFS camera & RETICON & RETICON & EBCCD & EBCCD & EBCCD & EBCCD & EBCCD \\
\hline IR wavelength & $2.12 \mu \mathrm{m}$ & $2.12 \mu \mathrm{m}$ & $2.15 \mu \mathrm{m}$ & $2.15 \mu \mathrm{m}$ & $2.15 \mu \mathrm{m}$ & $2.15 \mu \mathrm{m}$ & $2.15 \mu \mathrm{m}$ \\
\hline Total integration time & $20 \mathrm{~s}$ & $20 \mathrm{~s}$ & $50 \mathrm{~s}$ & $50 \mathrm{~s}$ & $100 \mathrm{~s}$ & $100 \mathrm{~s}$ & $100 \mathrm{~s}$ \\
\hline average gain & 0.56 & 0.46 & 0.19 & 0.49 & 0.09 & 0.08 & 0.27 \\
\hline gain min - gain $\max$ & $0.30-1.0$ & $0.33-0.69$ & $0.04-0.51$ & $0.09-0.89$ & $0.03-0.3$ & $0.1-0.26$ & $0.1-0.44$ \\
\hline Residual phase $\sigma_{\epsilon}^{2}$ at $0.55 \mu \mathrm{m}$ & $22.8 \mathrm{rd}^{2}$ & $21.1 \mathrm{rd}^{2}$ & $40.6 \mathrm{rd}^{2}$ & $47.4 \mathrm{rd}^{2}$ & $23.1 \mathrm{rd}^{2}$ & $20.9 \mathrm{rd}^{2}$ & $20.8 \mathrm{rd}^{2}$ \\
\hline$\hookrightarrow$ Low-order residual phase $\sigma_{\epsilon \|}^{2}$ & $18.6 \mathrm{rd}^{2}$ & $15.6 \mathrm{rd}^{2}$ & $30.8 \mathrm{rd}^{2}$ & $33.7 \mathrm{rd}^{2}$ & $19.9 \mathrm{rd}^{2}$ & $18.4 \mathrm{rd}^{2}$ & $17.6 \mathrm{rd}^{2}$ \\
\hline$\hookrightarrow$ High-order phase $\sigma_{\phi_{\mathrm{a}}}^{2}$ & $4.2 \mathrm{rd}^{2}$ & $5.5 \mathrm{rd}^{2}$ & $9.8 \mathrm{rd}^{2}$ & $13.7 \mathrm{rd}^{2}$ & $3.2 \mathrm{rd}^{2}$ & $2.5 \mathrm{rd}^{2}$ & $3.2 \mathrm{rd}^{2}$ \\
\hline Modal measurement error $\sigma_{\mathrm{n}}^{2}$ & $17.5 \mathrm{rd}^{2}$ & $28.6 \mathrm{rd}^{2}$ & $32.7 \mathrm{rd}^{2}$ & $40.7 \mathrm{rd}^{2}$ & $128.1 \mathrm{rd}^{2}$ & $179.8 \mathrm{rd}^{2}$ & $33.0 \mathrm{rd}^{2}$ \\
\hline Remaining error $\sigma_{\mathrm{r}}^{2}$ & $1.2 \mathrm{rd}^{2}$ & $1.5 \mathrm{rd}^{2}$ & $2.2 \mathrm{rd}^{2}$ & $3.1 \mathrm{rd}^{2}$ & $0.7 \mathrm{rd}^{2}$ & $0.6 \mathrm{rd}^{2}$ & $0.7 \mathrm{rd}^{2}$ \\
\hline Strehl ratio of observed PSF & $25.5 \%$ & $24.4 \%$ & $10.1 \%$ & $7.6 \%$ & $21.6 \%$ & $19.9 \%$ & $26.3 \%$ \\
\hline Strehl ratio of reconstructed PSF & $26.7 \%$ & $28.6 \%$ & $9.4 \%$ & $6.2 \%$ & $21.6 \%$ & $24.7 \%$ & $27.1 \%$ \\
\hline FWHM of observed PSF & 0.126 & 0.135 & 0.187 & 0.199 & 0.169 & 0.178 & 0.159 \\
\hline FWHM of reconstructed PSF & 0.129 & 0.126 & 0.174 & 0.170 & 0.160 & $0^{\prime \prime} 156$ & 0.162 \\
\hline
\end{tabular}

to 9 compare the observation (continuous line) to the reconstruction (dashed line).The left columns are the radial mean of the OTF in logarithmic scale. The central and right columns are the cuts of the PSF for two perpendicular directions in linear scale. The dotted lines represent the reconstruction error in linear scale.

\subsection{First examples: Bright reference sources and good seeing}

We observed a bright reference source with two slightly different neutral densities (7.4 et $7.7 \mathrm{mag})$ and under good seeing conditions ( 0.9 to 1 arcsec). The correction was good leading to observed Strehl ratios of about $25 \%$. Since we did not calibrate the non-common path aberrations, we took the ideal instrumental OTF for the reconstruction. The results are shown in Fig. 7. For both examples, the maximum reconstruction error on the OTF is about 10 to $20 \%$ at low frequencies. The maximum error on the PSF is located at the first-diffraction ring. In contrast to the reconstructed PSF, the observed one is not centrosymmetric. Note that this asymmetry has changed from one observed PSF to the other for the $x$-direction. Noncommon path aberrations, which we did not calibrate, can in principle produce an asymmetry in the PSF, but it is difficult to imagine how they could have changed on such a short time scale without moving the telescope (see also Sect. 5.2 and Fig. 17).

\subsection{Second examples: Faint reference sources and very bad seeing}

These examples were obtained with the EBCCD under very bad seeing conditions ( 1.5 to 2 arcsec). The magnitudes of the reference sources are between 10 and 12 and the observed Strehl ratios vary from 4 to $10 \%$. Since the correction was very partial, we estimated the measurement noise on the CL data. Figure 8 shows that, for both examples, the reconstructed OTF is underestimated by at least 20 to $30 \%$ at low frequencies. This was the case for each source, all faint, observed during the same night. The non-common path aberrations were calibrated by taking an image of a fiber source at the beginning of the night. However, we found that using either the ideal or the measured instrumental OTF does not change significantly the quality of the PSF reconstruction.

\subsection{Third examples: Faint sources and very good seeing}

The last examples correspond to reference sources of 10 to 12 magnitudes, obtained under very good seeing conditions $(0.6-0.7$ arcsec $)$. The observed Strehl ratios are 
Radial mean of OTF
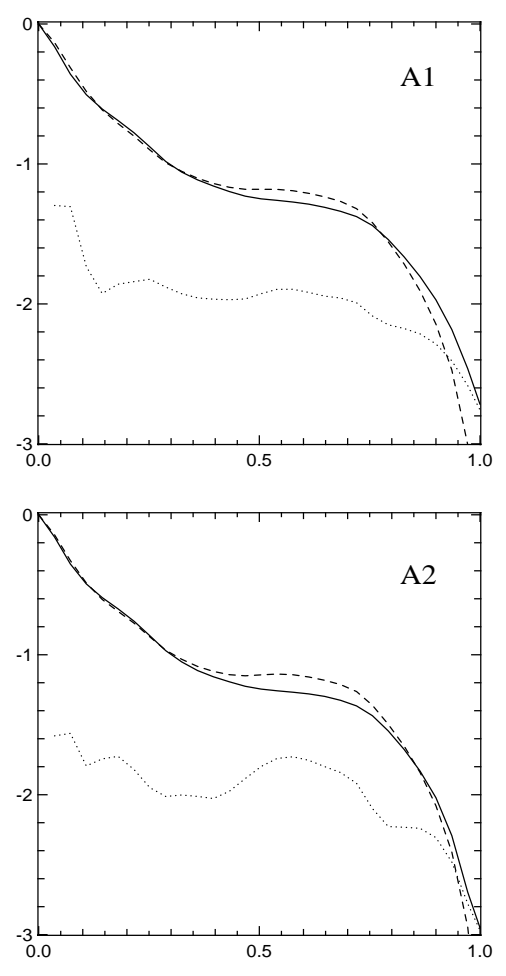

Normalized frequency
X cut of PSF
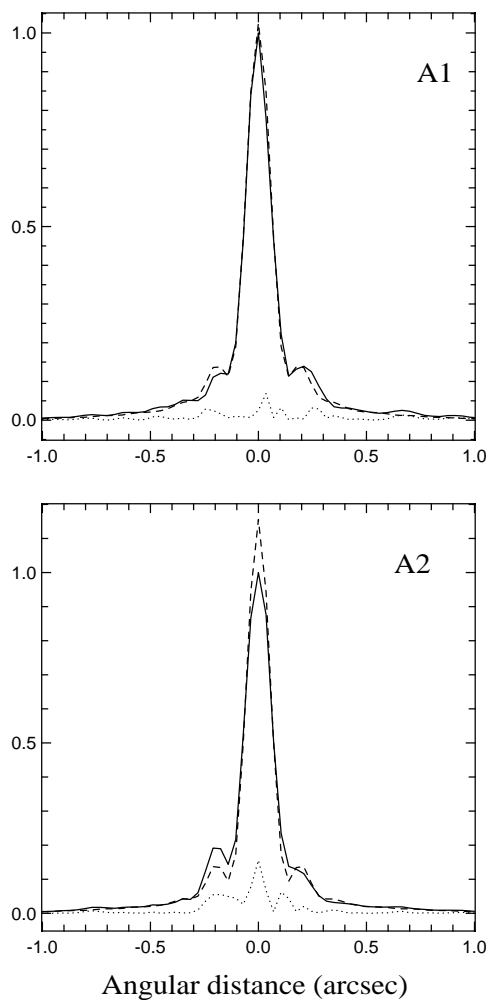

Y cut of PSF
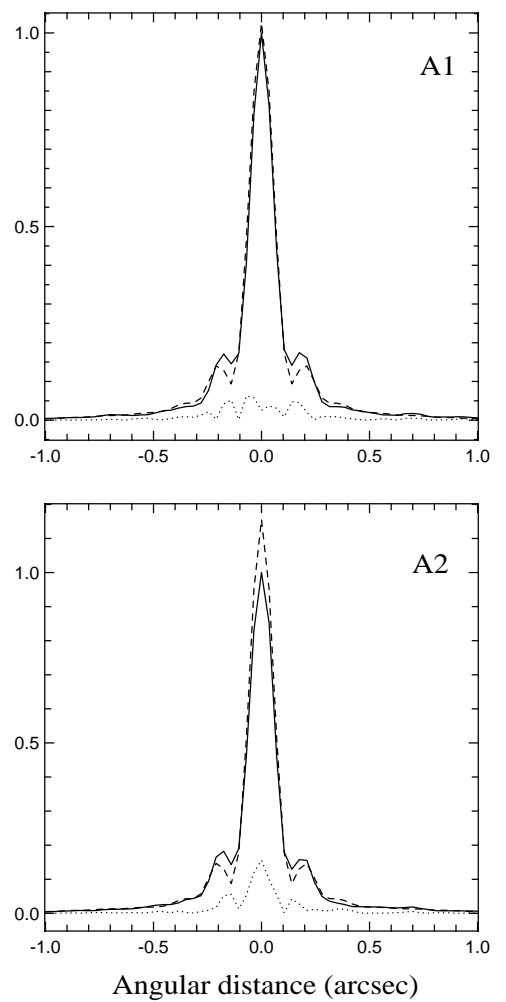

Fig. 7. Comparison between the observation (continuous line) and the reconstruction (dashed line) for examples A1 and A2. Left column: radial mean of the OTF in logarithmic scale. Central and right columns: cuts of the PSF for two perpendicular directions in linear scale. The dotted lines represent the reconstruction error in linear scale

between 20 and 30\%. Since the residual aberration visible on the first diffraction ring is stable from one image to another one, we calibrated $\mathrm{OTF}_{\text {sta }}$ by comparing the observed OTF of the same reference source taken before with its reconstructed OTF (Véran et al. 1997b).

The results are shown in Fig. 9. The error on the reconstructed OTF of examples C1 and C3 is less than 10\% at low and medium frequencies. The asymmetry of the PSF is quite well reproduced within a mean error between 5 and $10 \%$. In example $\mathrm{C} 2$, the reconstruction error is much larger. In this case, the average measured flux per sub-aperture on the WFS is about 4.5 photoelectrons. A closer look to the corresponding set of measurements reveals, from time to time, fluxes of just 2, 1 or even no photoelectrons at all. In these cases the center of gravity on the WFS is poorly estimated.

\section{Discussion}

For good seeing and good correction, the OTF is well estimated at low frequencies (see examples $\mathrm{C} 1$ and $\mathrm{C} 3$, and to a less extent, examples A1 and A2). The reconstruction, however, is severely limited by the presence of a residual aberration visible on the first diffraction ring. This aberration which cannot be reproduced by our model can be quite variable. In the case of very bad seeing and very partial correction (examples B), the OTF is always largely underestimated. In this section, we discuss the possible error sources in the PSF reconstruction process and we examine the consequences on the photometric precision we can reach with the ADONIS system.

\subsection{Temporal bandwidth}

When computing the covariance matrix of the residual modal coefficients $\mathcal{C}_{\epsilon \epsilon}$ and when estimating $r_{0}$ from the variance of the mirror commands, we assume that the bandwidth of the AO system is higher than $\nu_{\mathrm{c}}$, the highest cut-off frequency of the turbulent modes (see Sect. 2.3.1). As seen in Sect. 2.2.2, the bandwidth of the AO system depends on the corrected mode via its associated gain. The mean bandwidth of ADONIS is typically about $10 \mathrm{~Hz}$. The highest cut-off frequency of the turbulent modes analyzed by the system (mirror modes) corresponds roughly to ${ }^{4}$

$\nu_{\mathrm{c}} \approx 0.3(n+1) \frac{V}{D} \approx 8-9 \mathrm{~Hz}$.

\footnotetext{
4 Assuming Kolmogorov turbulence, Taylor hypothesis with a wind speed $V$ of $10 \mathrm{~m} / \mathrm{s}$ and Zernike degree $n=9$ (Conan et al. 1995).
} 
Radial mean of OTF
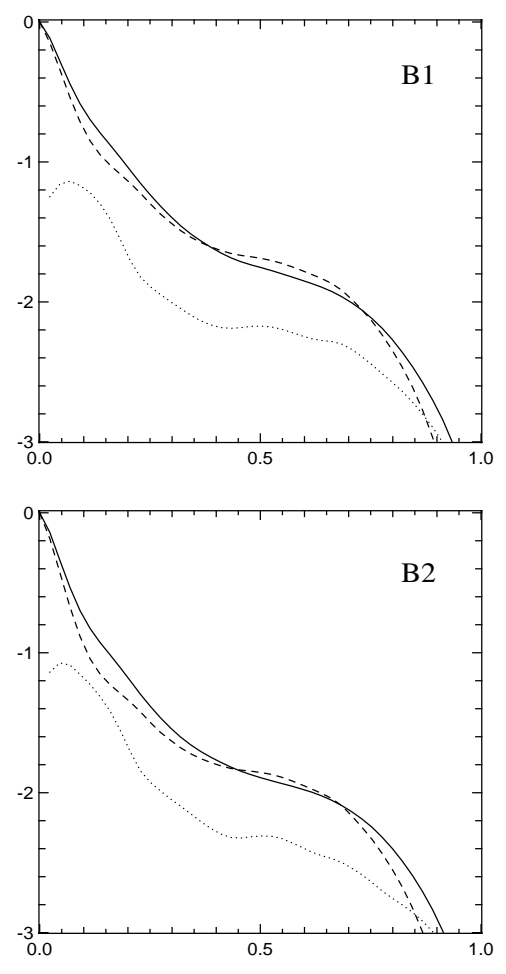

Normalized frequency
$\mathrm{X}$ cut of PSF
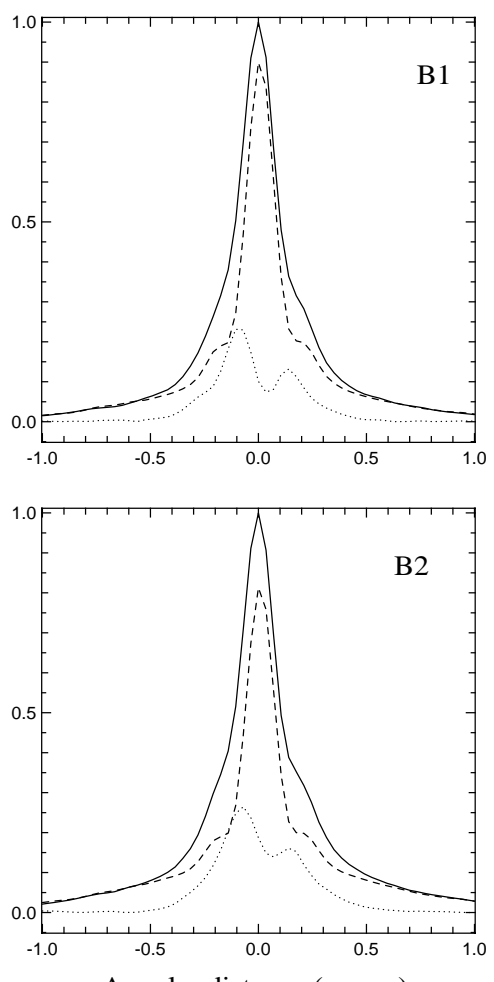

Angular distance $(\operatorname{arcsec})$
Y cut of PSF
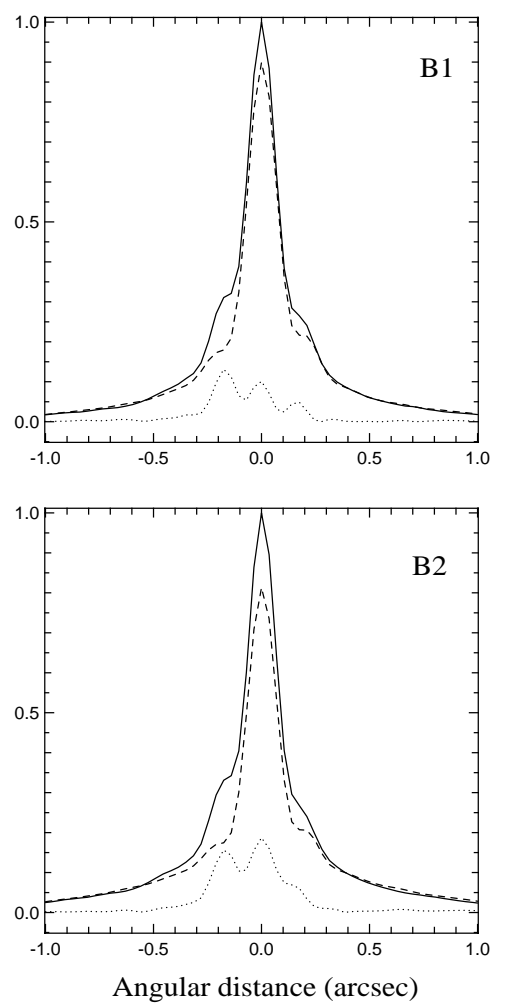

Fig. 8. Comparison between the observation (continuous line) and the reconstruction (dashed line) for examples B1 and B2. Same legends as in Fig. 7

Clearly, the hypothesis of a large bandwidth is not quite well justified. Hence, it may not be justified to set the term

$$
\begin{aligned}
\mathcal{E}_{i j}= & -\int H_{\text {cor }}\left(g_{i}, \nu\right)\left\{S_{\mathrm{a}_{i} \mathrm{r}_{j}}(\nu)+S_{\mathrm{r}_{i} \mathrm{r}_{j}}(\nu)\right\} \mathrm{d} \nu \\
& -\int H_{\text {cor }}\left(g_{j}, \nu\right)\left\{S_{\mathrm{a}_{j} \mathrm{r}_{i}}(\nu)+S_{\mathrm{r}_{i} \mathrm{r}_{j}}(\nu)\right\} \mathrm{d} \nu
\end{aligned}
$$

equal to 0 in Eq. (25). It is difficult to evaluate it precisely. In order to derive an order of magnitude of its maximum value, we simply assume that $S_{\mathrm{ar}}$ is similar to $S_{\mathrm{rr}}$ and that the transfer function $H_{\text {cor }}$ just filters out half of the total energy $\left(H_{\text {cor }}=\frac{1}{2}\right)$. In this case, since $\left\langle r_{i} r_{j}\right\rangle=$ $\int S_{\mathrm{r}_{i} \mathrm{r}_{j}}(\nu) \mathrm{d} \nu$, we find $\mathcal{E} \approx-2 \mathcal{C}_{r r}$. Figure 10 shows, for example B1, the OTF calculated with $\mathcal{E}=0$ (continuous line) and the OTF calculated with $\mathcal{E}=-2 \mathcal{C}_{r r}$ (dashed line). None of the both cases can explain the experimental results (dotted line).

For low bandwidths, the estimated $r_{0}$ may also be biased leading to a value larger than the true one (see Sect. 3.2) and thus to a Strehl ratio which is larger than the experimental one. However, the Strehl ratios of the estimated PSFs in examples B are smaller than those of the observed PSFs. In addition, when calculating $r_{0}$ from the high-order mirror modes, we find even smaller values than from the low-order mirror modes. All these results prove that the large reconstruction errors in case $\mathrm{B}$ cannot be explained by a too small bandwidth of the AO system. We have no clear explanation for the failure of the reconstruc- tion in case of bad seeing. It may be due to the presence of speckles on the WFS image which induce biases in the calculation of the center of gravity. Simulations may help to verify this hypothesis.

\subsection{Local turbulence}

The expression for the long-exposure optical transfer function in Eq. (22) assumes that the residual phase is a random variable of Gaussian statistics. As a consequence, the atmospheric residual term in Eq. (22), given by the product $\exp -\frac{1}{2} \bar{D}_{\phi_{\epsilon_{\|}}} \times \exp -\frac{1}{2} \bar{D}_{\phi_{\epsilon_{\perp}}}$, is real and the corresponding PSF therefore centro-symmetric. The only way to produce in our model a complex transfer function is through the instrumental term $\mathrm{OTF}_{\text {sta }}$, via non-common path aberrations which are assumed to be static.

Figure 17 shows two series of 10 images of 2 second integration time each and the corresponding longexposure images (sum of the short-exposure images). A residual aberration is visible on the first diffraction ring. It varies randomly in intensity and direction and hence its origin cannot be due to non-common path aberrations. Furthermore, it is non-stationary and seems to evolve slowly compared to the deformations produced by an atmospheric turbulence. We therefore believe that its origin 
S. Harder and A. Chelli: Estimating the point spread function of the adaptive optics system ADONIS

Radial mean of OTF
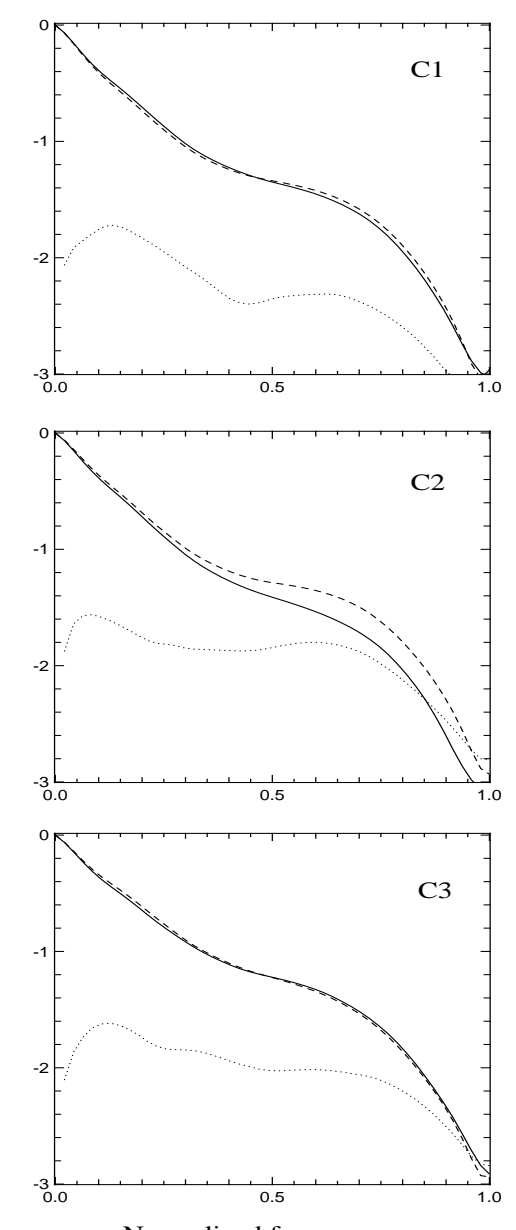

$\mathrm{W}$
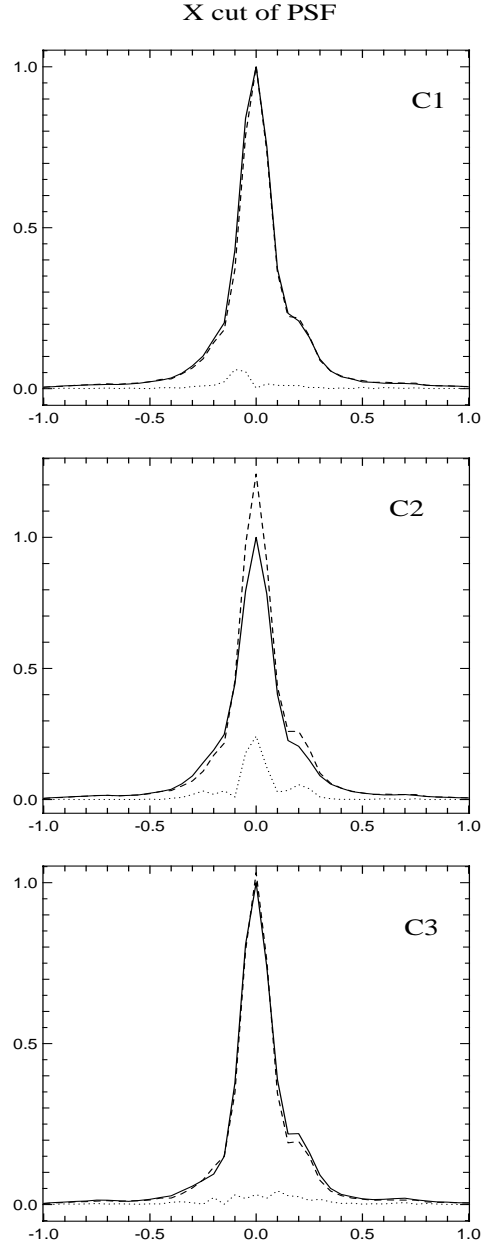

Angular distance $(\operatorname{arcsec})$
Y cut of PSF
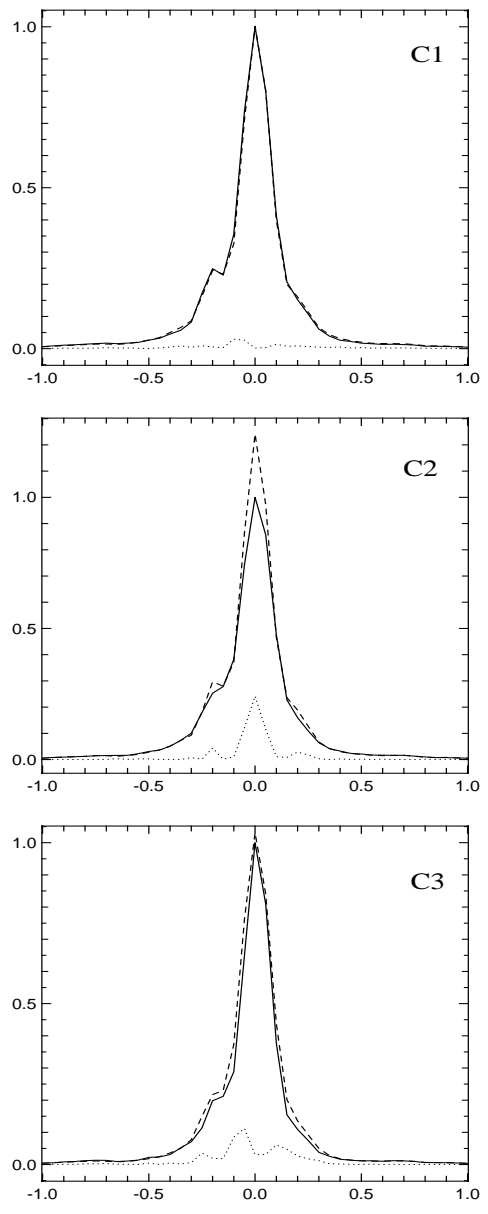

Angular distance $(\operatorname{arcsec})$

Fig. 9. Comparison between the observation (continuous line) and the reconstruction (dashed line) for examples C1, C2 and C3. Same legends as in Fig. 7

Table 3. Values of $r_{0}(\mathrm{~cm})$ estimated from the variance of angle of arrival in OL $\left(r_{0_{\mathrm{OL}}}\right)$, from the modal mirror commands 3 to $5\left(r_{0_{3-5}}\right), 6$ to $9\left(r_{0_{6-9}}\right)$ and 10 to $25\left(r_{0_{10-25}}\right)$

\begin{tabular}{lccccc}
\hline \hline PSF & $r_{0_{\mathrm{OL}}}$ & $r_{0_{3-5}}$ & $r_{0_{6-9}}$ & $r_{0_{10-25}}$ & $r_{0_{25-50}}$ \\
\hline C1 (Oct 98) & 17.8 & 17.7 & 15.1 & 11.8 & 8.6 \\
C2 & 16.4 & 16.8 & 13.9 & 12.4 & 8.5 \\
C3 & 20.3 & 18.7 & 16.0 & 14.2 & 11.4 \\
C4 & 18.8 & 19.0 & 15.8 & 12.3 & 8.1 \\
B1 (May 98) & & 9.2 & 7.7 & 6.7 & 6.0 \\
B2 & & 7.5 & 6.4 & 5.4 & 5.0 \\
Dec. 98 & & 11.2 & 10.1 & 8.0 & 6.9 \\
\hline \hline
\end{tabular}

is due to the presence of a local turbulence, situated e.g. above the primary mirror.

Further support for this hypothesis comes from the comparison of the variance of the mirror commands $\sigma_{\mathrm{m}_{i}}^{2}$ with the variances $\sigma_{\mathrm{a}_{i}}^{2}+\sigma_{\mathrm{r}_{i}}^{2}$ predicted by the theory of Kolmogorov. Figures $11 \mathrm{a}$ and $\mathrm{b}$ show,

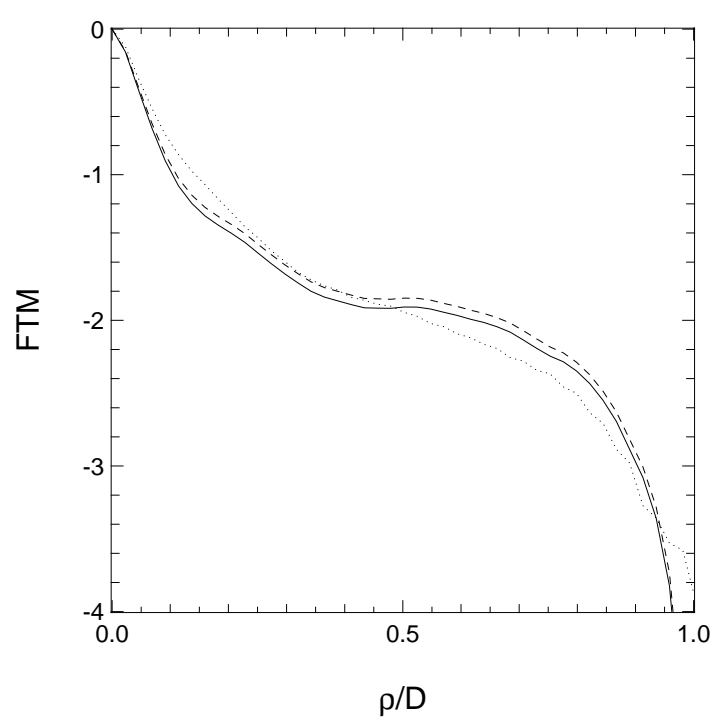

Fig. 10. The continuous line shows the reconstructed OTF assuming a high bandwidth and the dashed line the reconstructed OTF for a low bandwidth (see text). The dotted line shows the observed OTF 

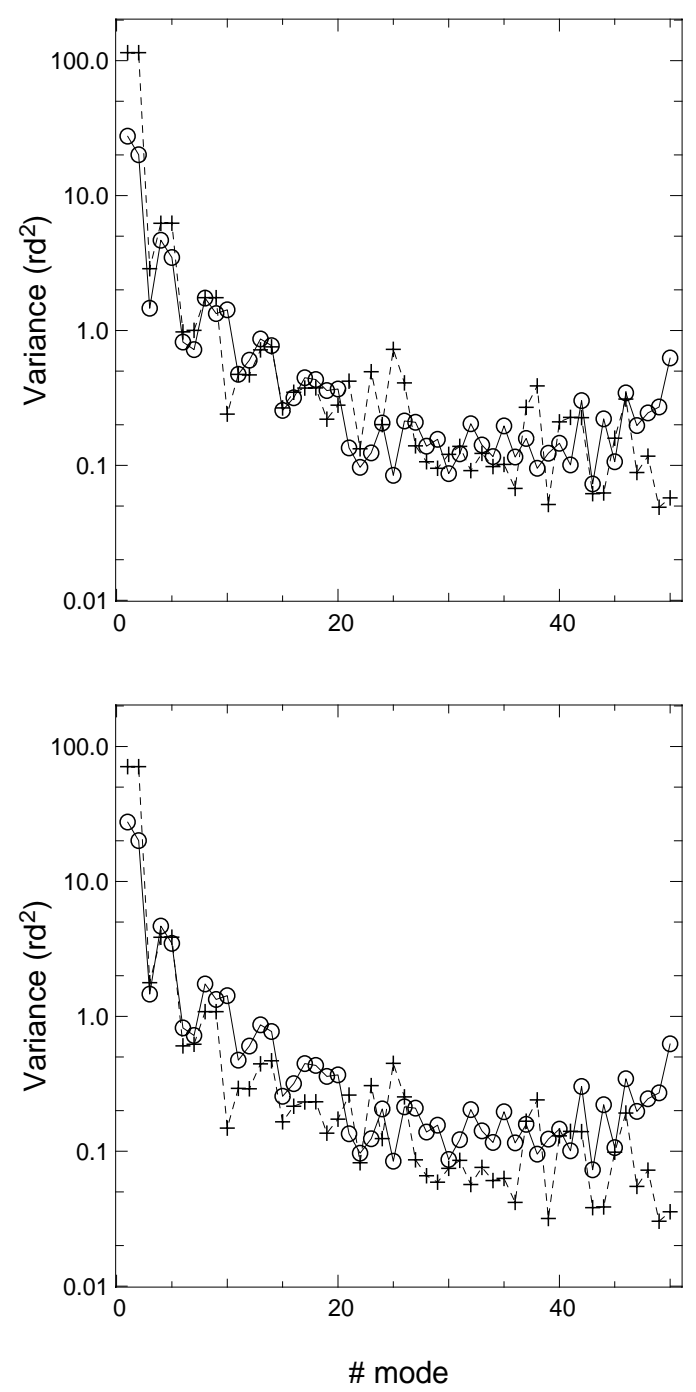

Fig. 11. Variance of the mirror commands $\sigma_{\mathrm{m}_{i}}^{2}$ (continuous line + circles) and variance of the turbulent modes $\sigma_{\mathrm{a}_{i}}^{2}+\sigma_{\mathrm{r}_{i}}^{2}$ (dashed line) as a function of the mode $i$, assuming a) $r_{0}=14 \mathrm{~cm}$ and b) $r_{0}=19 \mathrm{~cm}$

in logarithmic scale, $\sigma_{\mathrm{m}_{i}}^{2}$ (continuous line + circles) compared to $\sigma_{\mathrm{a}_{i}}^{2}+\sigma_{\mathrm{r}_{i}}^{2}$ (dashed lines + crosses) as a function of the mode number $i$, for $r_{0}=14 \mathrm{~cm}$ and $r_{0}=$ $19 \mathrm{~cm}$, respectively. In the first case, there is a good agreement between both variances for the modes 11 to 20 , while in the second case the agreement is good for the modes 3 to 5 . Note that there is an important deviation from the theory for the mode 10 and for several higher modes $(>25)$. In Table 3 , we present the values of $r_{0}$ obtained when fitting the turbulent variance to the mirror variance on different groups of modes: 3 to $5\left(r_{0_{3-5}}\right), 6$ to $9\left(r_{0_{6-9}}\right), 10$ to $25\left(r_{0_{10-25}}\right)$ and 26 to $50\left(r_{0_{26-50}}\right)$. The calculated $r_{0}$ decrease with the degree of the modes.

This trend cannot be explained by the small bandwidth of the AO system, because we would observe the opposite effect, i.e. an increasing $r_{0}$ with the mode number

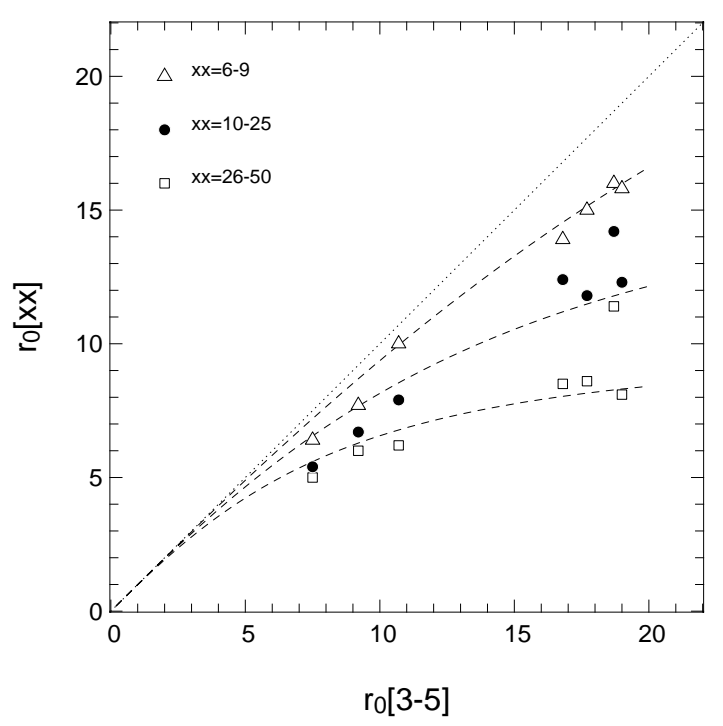

Fig. 12. Estimated values of $r_{0}$ (from Table 3$): r_{0_{6-9}}, r_{0_{10-25}}$, $r_{0_{26-50}}$ versus $r_{0_{3-5}}$. The dashed lines represent the predicted estimated value of $r_{0}$ biased by the presence of a local turbulence (see text)

(see Sect. 3.2). It can neither be explained by the effect of the outer turbulence scale $L_{0}$. Indeed, $r_{0}$ estimated from the variance of the angle-of-arrival, taking into account $L_{0}$, coincides with $r_{0}$ estimated from the variance of the mirror modes 3 to 5 , but not taking into account $L_{0}$ (see Sect. 3.2 and Table 3 ). In addition, the influence of $L_{0}$ decreases as the mode number increases.

On the other hand, the observed trend of the calculated $r_{0}$ to decrease with the mode number, can well be explained by the presence of a local turbulence. $r_{0_{3-5}}$ being consistent with other independent estimations, we assume that only the modes superior to 5 are affected by the local turbulence and that its contribution $\sigma_{1}^{2}$ to the variance is independent of the mode:

$$
\begin{aligned}
& \sigma_{\text {turb }_{i}}^{2}=\sigma_{\mathrm{a}_{i\left(D / r_{0}=1\right)}}^{2}\left(\frac{D}{r_{0}}\right)^{5 / 3} \text { for } i \leq 5 \\
& \sigma_{\text {turb }_{i}}^{2}=\sigma_{\mathrm{a}_{i\left(D / r_{0}=1\right)}}^{2}\left(\frac{D}{r_{0}}\right)^{5 / 3}+\sigma_{1}^{2} \text { for } i>5,
\end{aligned}
$$

where $r_{0}$ is the true value of the atmospheric turbulence $\sigma_{\mathrm{a}}^{2}$. With these hypotheses $r_{0_{i}}$ calculated for the modes superior to 5 is given by:

$$
r_{0_{i}}=\frac{D}{\left[\left(\frac{D}{r_{0}}\right)^{5 / 3}+\frac{\sigma_{1}^{2}}{\sigma_{a_{(}\left(D / r_{0}=1\right)}^{2}}\right]^{3 / 5}} .
$$

From this equation, we clearly see that $r_{0_{i}}$ decreases as the atmospheric seeing decreases $\left(\frac{D}{r_{0}}\right.$ increases $)$ and also as the mode number increases ( $\sigma_{\mathrm{a}_{i}}^{2}$ decreases). Figure 12 shows, for different observing conditions, the apparent $r_{0}$ as a function of $r_{0_{3-5}}$. The three dashed lines representing the model given by Eq. (33) with a single $\sigma_{1}^{2}$-value 

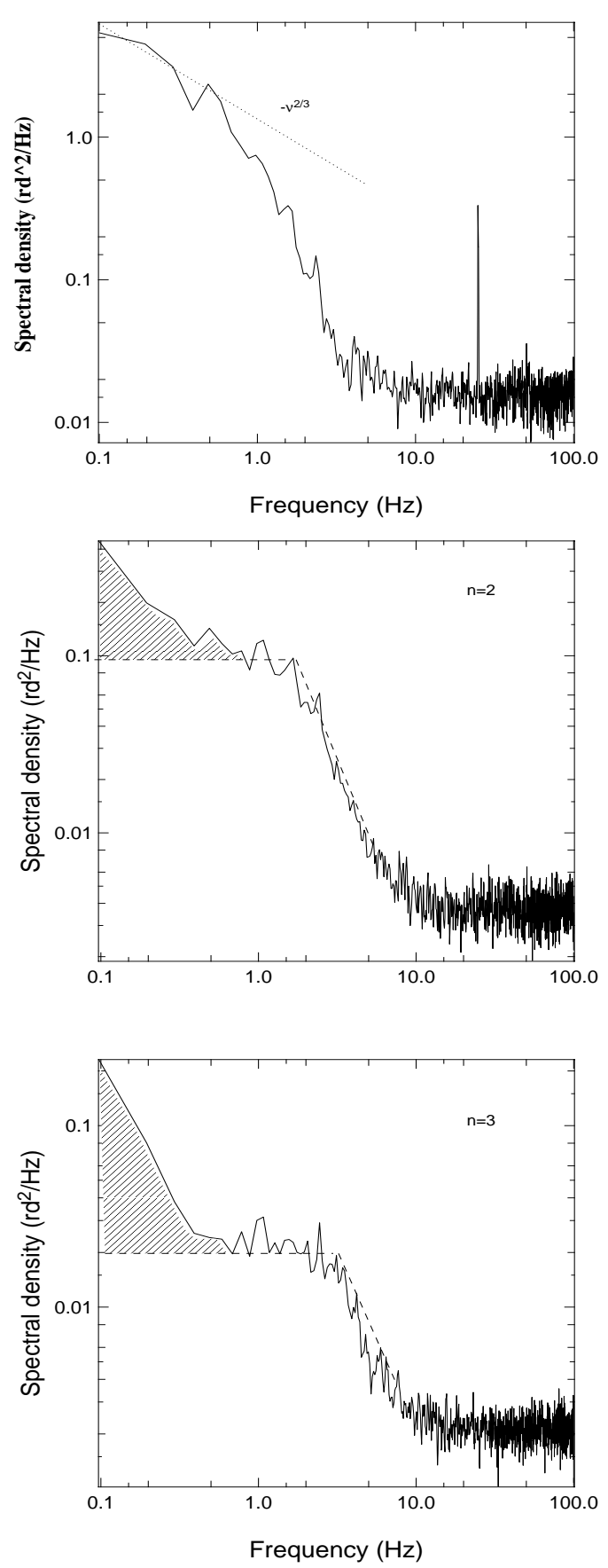

Fig. 13. Temporal spectral densities for a) $n=1$ (tip/tilt), b) $n=2$ (defocus/astigmatism) and c) $n=3$ (coma/triangular coma). The dashed lines show the spectra predicted from the Kolmogorov model and the Taylor hypothesis (see text)

fit quite well the results. The total contribution of the local turbulence to the whole mirror modes is about $10 \mathrm{rd}^{2}$, which corresponds to $7-8 \%$ of the total contribution of the atmospheric turbulence for $r_{0}=19 \mathrm{~cm}$.

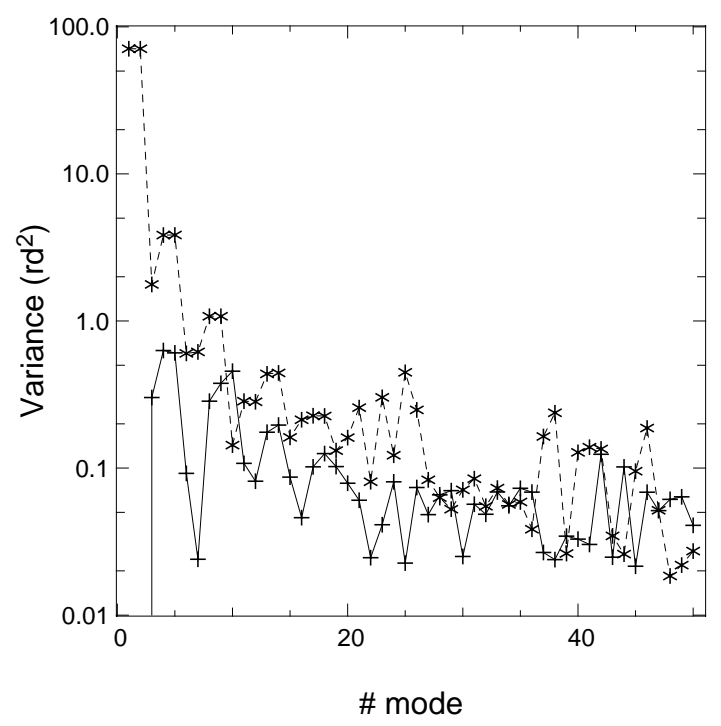

Fig. 14. Variance of the local turbulence $\sigma_{1_{i}}^{2}$ (continuous line) and the variance of the Kolmogorov turbulence $\sigma_{\mathrm{a}_{i}}^{2}$ for $r_{0}=$ $19 \mathrm{~cm}$ (dashed line) in function of the mode $i$

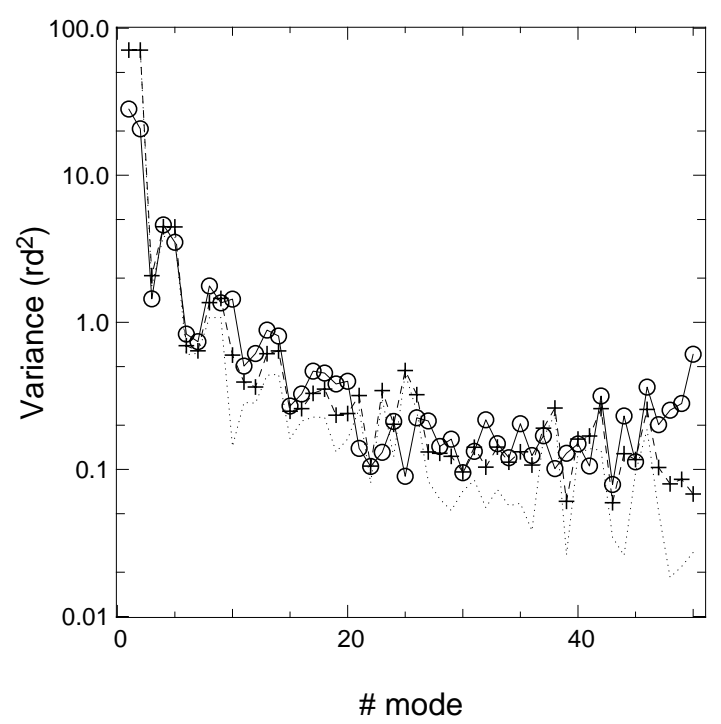

Fig. 15. Variance of the mirror commands $\sigma_{\mathrm{m}_{i}}^{2}$ (continuous line) and the sum of the variance of the local turbulence and the atmospheric turbulence $\sigma_{\mathrm{a}_{i}}^{2}+\sigma_{1_{i}}^{2}$ (dashed line) in function of the mode $i$. We also show the variance of the atmospheric turbulence $\sigma_{\mathrm{a}_{i}}^{2}$ (dotted line)

To have a better insight into the physical properties of this local turbulence, we calculate the temporal spectral densities of the turbulent modes. They are shown in Figs. 13a-c. Figure 13a presents the average power spectrum for the first two modes (tip/tilt). At very low frequencies, it is proportional to $\nu^{-2 / 3}$ as predicted from the Kolmogorov model and the Taylor hypothesis (Conan et al. 1995). Figures $13 \mathrm{~b}$ and $\mathrm{c}$ show the average power spectrum for the modes 3 to 5 (defocalisation and astigmatism) and the average power spectrum for the modes 6 to 9 (coma and triangular coma), respectively. In both 

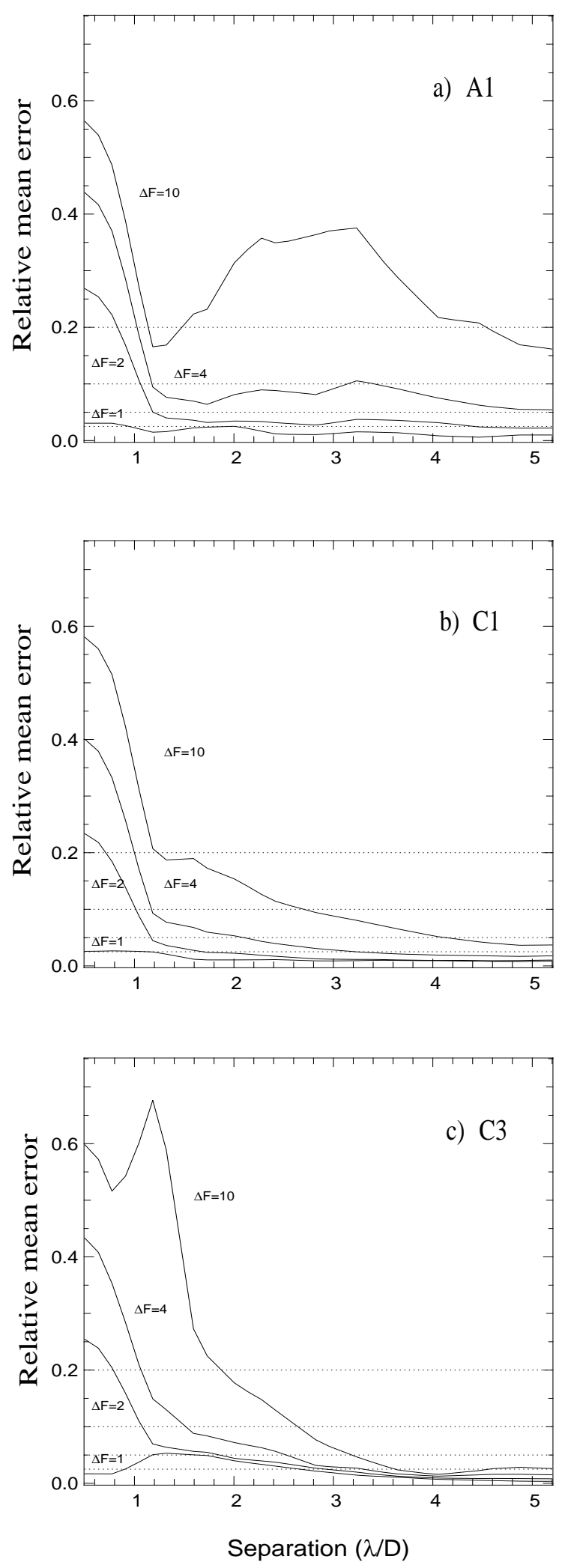

Fig. 16. Mean relative error on the flux ratio versus the separation of the sources for flux ratios 1,2, 4 and 10, for a) example $\mathrm{A} 1$, for $\mathbf{b}$ ) example $\mathrm{C} 1$ and for $\mathbf{c}$ ) example $\mathrm{C} 3$ figures, the dashed lines represent the predicted mean spectra, when assuming a frozen Kolmogorov turbulence and a wind speed of typically $12 \mathrm{~m} / \mathrm{s}$ (Conan et al. 1995). This theoretical spectra are constant at low frequencies and decrease as $\nu^{-12 / 3}$ after a cut-off frequency of about 3 to $4 \mathrm{~Hz}$. The two observed spectra clearly show the presence of a turbulent component (grey area) with a cut-off frequency of about $0.5 \mathrm{~Hz}$ which cannot be of atmospheric origin. We observe this component for the spectra of all modes superior to 2 . It proves the existence of a local turbulence, probably located above the primary mirror.

From the temporal spectra, we can estimate the contribution of the local turbulence to the variance of the mirror modes. Figure 14 shows this contribution and that of the atmospheric turbulence for $r_{0}=19 \mathrm{~cm}$. In this case, i.e. good seeing, they are comparable at high order modes. The total contribution of the local turbulence to the mirror modes is found to be about $7 \mathrm{rd}^{2}$, in very good agreement with the value derived from the $r_{0}$ analysis $\left(10 \mathrm{rd}^{2}\right)$. Note that: i) for the modes 3 to 5 , the force of the local turbulence is much lower than that of the atmospheric turbulence, and ii) for the modes superior to 5 , to a first approximation, it is constant. This validates a posteriori the hypotheses done in Eq. (32). Figure 15 shows the variance of the mirror commands $\sigma_{\mathrm{m}_{i}}^{2}$ (continuous line + circles $)$ and $\sigma_{\mathrm{a}_{i}}^{2}+\sigma_{\mathrm{l}_{i}}^{2}\left(\sigma_{\mathrm{r}_{i}}^{2}\right.$ is negligible in the present case), the sum of the variances of the atmospheric and the local turbulence (dashed line + crosses). Clearly, $\sigma_{\mathrm{m}_{i}}^{2}$ is well fitted when taking into account the term $\sigma_{\mathrm{l}_{i}}^{2}$, in particular the mode number 10 is much better reproduced (compare also with Fig. 11).

We have seen that the residual low-order aberration not reproduced by the reconstruction model can be very variable (examples A) or quite stable (examples $\mathrm{C}$ ). In this section, we interpret the residual aberration in the corrected image, at least its variable part, as the effect of a local turbulence. Why does the system not correct this low-order aberration efficiently, since the cut-off frequency is found to be much lower than the correction bandwidth? We have no definite answer to this question. However, the problem may be due to the influence of the local turbulence on the remaining error, i.e. the contribution of the high-frequency phase $\phi_{\perp}$ to the residual phase. Indeed, the remaining error is always present, and its relative contribution to the residual phase becomes even more important, and hence more visible, when the correction is very good, i.e. when the WFS noise is very small.

We conclude that the residual aberration visible on the first diffraction ring is not necessarily a consequence of a bad low-order correction of the local turbulence, but can be due to the presence of a remaining error highly affected by the local turbulence. It is worth noting that the phenomenon of a variable residual aberration seems not to be restricted to the ADONIS system. Apparently (from private communications), it has also been 
Example A1
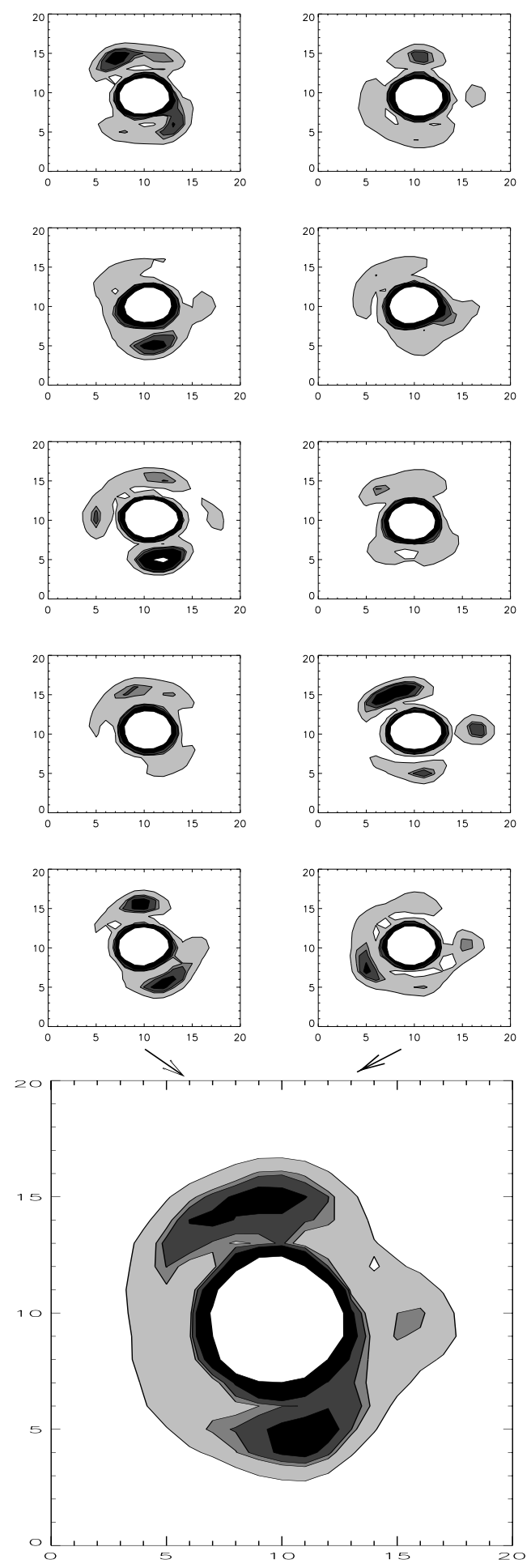

Example A2
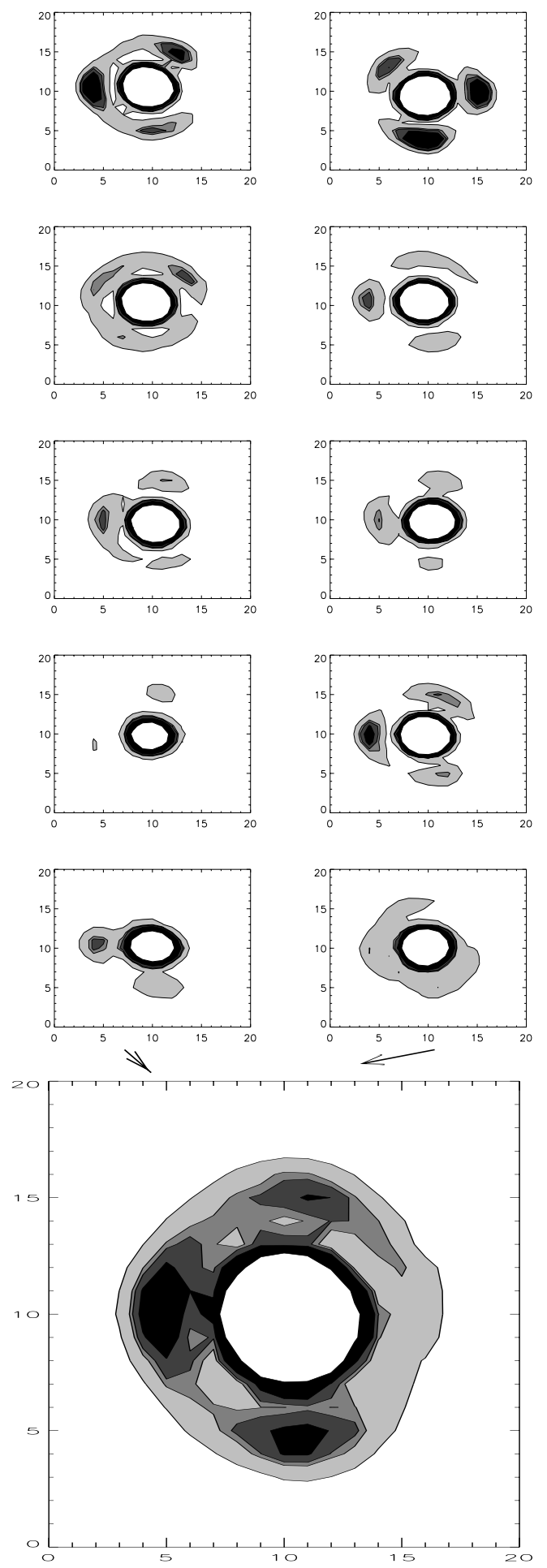

Fig. 17. Contour plots of the observed PSFs for a) example A1 and b) example A2. Each image at the bottom is the sum of the 10 images of $2 \mathrm{~s}$ integration time shown above it. The two examples represent the acquisition of the same reference source separated by an interval of about 10 to 20 minutes 
occasionally observed on the PUEO system (curvature sensor), but with a fainter magnitude.

\subsection{Photometric precision}

In this section, we investigate the photometric precision we can expect when deconvolving science exposures with the reconstructed PSF. For simplicity, we simulate the image of a binary system by convolving two point sources, having flux ratios of 1, 2, 4 and 10, with an observed PSF. We consider 8 equidistant position angles, and we vary the separation between the two sources from 0.6 to $5 \lambda / D$. Then, we deconvolve each image with the reconstructed PSF using the Lucy-Richardson algorithm. Since the two sources are well separated after deconvolution, we calculate the flux by integrating within an appropriated box centered on each source. Figures 16a-c show the relative error on the calculated flux ratio, averaged over all directions, versus the separation of the sources, for example A1 for which we could not calibrate the residual aberration and for examples $\mathrm{C} 1$ and $\mathrm{C} 3$ for which the residual aberration was calibrated.

Without this calibration the error on the calculated flux ratio is quite large at the location of the first diffraction ring (40\% for flux ratios of 10 and $10 \%$ for flux ratios of 4) and remains important even at large distances $(5-15 \%)$. When the residual aberration can be calibrated, the error is smaller. For flux ratios larger than 4 , it is of the order of $3 \%$ at large distances, but it increases to about $5-10 \%$ at the location of the first diffraction ring which indicates that the residual aberration has slightly varied from one long exposure image to another. For flux ratios between 1 and 2, the error is less than $3 \%$ at all distances.

The error on the flux ratio depends on our exact knowledge of the object's PSF at the time of its acquisition. When reconstructing the PSF from the AO data, this knowledge seems to be essentially limited, at least under good seeing conditions, by the variations of the residual aberration visible on the first diffraction ring. When using an experimental PSF, this knowledge is also limited by the degree of stationarity of the atmospheric turbulence. In order to compare these two approaches, we deconvolve the binary images (simulated with the observed PSF A1) with the observed PSF A2. The errors on the flux ratios are the same than those obtained with the reconstructed PSF. However, the PSF A1 and A2 were obtained on the same star, which is never the case for real observations, and have (by chance?) similar Strehl ratios. Rigaut \& Sarazin (1998) predict an uncertainty of $20 \%$ on the Strehl ratio, when using only one PSF calibration. In order to obtain an uncertainty of $2 \%$ on the Strehl ratio, one has to do about 100 round trips between the PSF and the science object acquisition. This clearly shows the importance of PSF reconstruction from $\mathrm{AO}$ data obtained simultaneously to the object image which allows to optimize telescope time and to reach higher photometric precisions. The Strehl ratio is not a sufficient criteria to describe the quality of the PSF as it is well demonstrated by examples A1 and A2 which have essentially the same Strehl ratio, but differ considerably on the first diffraction ring.

\section{Summary}

We reconstructed point spread functions of the ADONIS adaptive optics system from its wavefront sensor measurements. For very bad seeing (at least 1.5 arcsec), the PSF reconstruction is not satisfying. The OTF is always underestimated by 20 to $30 \%$ at low and medium frequencies. This discrepancy may be due to the presence of speckles on the WFS image which induce biases in the calculation of the center of gravity.

For good seeing and good correction, we estimate the OTF within $10 \%$ accuracy at low and medium frequencies. In this case, the major limitation is the presence of a residual non-modeled aberration visible on the first diffraction ring. This aberration is non-stationary and cannot be due to non-common path aberrations which are static.

The temporal spectra of the turbulent phase modes (for $i>2$ ) clearly prove the existence of a slowly evolving and non fully developed turbulence. This turbulence is probably due to temperature gradients above the primary mirror. It could be the cause of the residual aberration through the contamination of the estimated residual mirror phase by the high-order non-corrected modes.

This residual aberration limits the photometric precision we can expect on the ADONIS system. When the aberration is variable, the error on the flux ratio of a binary system is important. For flux ratios larger than 4, it is of the order of $10-40 \%$ at the location of the first diffraction ring and 5-15\% for larger distances. When it is stable, the error is much smaller, of the order of $3-10 \%$.

Reconstructing the PSF from AO data obtained simultaneously to the object image allows to optimize telescope time and to reach higher photometric precisions. It is therefore important to pursue the PSF reconstruction method. However, until the source of the local turbulence has not been identified and removed, doing accurate photometry and detecting faint structures without ambiguity on images obtained with ADONIS will be difficult.

Acknowledgements. We wish to thank F. Marchis, D. Le Mignant, O. Marco, D. Bonaccini and Jean-Luc Beuzit for providing us with the observational data. We thank G. Rousset for his useful comments, E. Gendron for his help to get the necessary information about ADONIS and J.P. Véran for clarifying discussions about the PSF reconstruction. 


\section{References}

Conan J.M., Rousset G., Madec P.Y., 1995, JOSA A, 12 (7), 1559

Conan J.M., 1995, Ph.D. Thesis, Université Paris XI, Orsay, France

Gendron E., Léna P., 1994, A\&A 291, 3377

Gendron E., Léna P., 1995, AASS 111, 153

Dai G.M., 1996, JOSA A 13 (6), 1218

Hermann J., 1981, JOSA 71 (8), 989

Noll R.-J., 1976, JOSA 66, 207

Rigaut F., Arsenault R., Kerr J., Salmon D., Northcott N., 1994, SPIE, Vol. 2201

Rigaut F., Sarazin M., 1998, in "Astronomy with adaptive optics", ESO/OSA topical meeting, Bonaccini D. (ed.)
Roddier F., 1981, in Progress in Optics, Vol. XIX, Wolf E. (ed.). North-Holland, Amsterdam, p. 281

Rousset G., 1994, in "Adaptive Optics for Astronomy", Alloin D.M. and Mariotti J.-M. (eds.), Vol. 423 of NATO Advanced Science Institutes Series. Kluwer Academic Publishers, p. 115

Southwell W.H., 1982, Proc. SPIE 365, 97

Véran J.P., 1997, Ph.D. Thesis, École Nationale Supérieure des Télécommunications, France

Véran J.P., Rigaut F., Maître H., Rouan D., 1997, JOSA A 14 (11)

Véran J.P., Rigaut F., Maître H., Rouan D., 1997, SPIE

Wang J., Markey J., 1978, JOSA 68 (1), 78

Ziad A., 1993, Ph.D. Thesis, Université de Nice Sophia Antipolis, France 